LBNL-53578

SCMAG-823

\title{
Cryogenic Refrigeration Requirements for Superconducting Insertion Devices in a Light Source
}

\author{
Michael A. Green \\ Lawrence Berkeley National Laboratory
}

\begin{abstract}
This report discusses cryogenic cooling superconducting insertion devices for modern light sources. The introductory part of the report discusses the difference between wiggler and undulators and how the bore temperature may affect the performance of the magnets. The steps one would take to reduce the gap between the cold magnet pole are discussed.

One section of the report is devoted to showing how one would calculate the heat that enters the device. Source of heat include, heat entering through the vacuum chamber, heating due to stray electrons and synchrotron radiation, heating due to image current on the bore, heat flow by conduction and radiation, and heat transfer into the cryostat through the magnet leads.

A section of the report is devoted to cooling options such as small cryo-cooler and larger conventional helium refrigerators. This section contains a discussion as to when it is appropriate to use small coolers that do not have J-T circuits. Candidate small cryo-coolers are discussed in this section of the report. Cooling circuits for cooling with a conventional refrigerator are also discussed.

A section of the report is devoted to vibration isolation and how this may affect how the cooling is attached to the device. Vibration isolation using straps is compared to vibration isolation using helium heat pipes. The vibration isolation of a conventional refrigeration system is also discussed.

Finally, the cool down of an insertion device is discussed. The device can either be cooled down using liquid cryogenic nitrogen and liquid helium or by using the cooler used to keep the devices cold over the long haul.
\end{abstract}




\section{INTRODUCTION}

This report discusses options for cooling superconducting insertion devices for modern light sources. At least three types of superconducting insertion devices are found on modern light sources. They are superconducting bending magnets, superconducting wigglers, and superconducting undulators.

Bending magnets can be considered as insertion devices that are an integral part of the machine lattice. Bending magnets are different in that a light source will not produce any synchrotron light if any of its bending magnets are not operable. Because a light source ring and all of its experiments can not operate if a superconducting bending magnet fails, the cryogenic refrigeration for a superconducting bending magnet must be extremely reliable. The Superbend dipoles on the ALS at the Lawrence Berkeley Laboratory are an example of the first type of insertion device [1]. These magnets are operating successfully on the ALS ring without affecting the quality of the light beams coming from the machine. In general, this report will not discuss cryogenic cooling of this type of insertion device. The cooling issues for the other types of devices apply to simple bending magnets as well.

Wiggers and undulators, on the other hand, often do not affect the beam dynamics of the machine in a large way. In other words, most light sources will operate if the field is turned off in a wiggler or undulator. However, it should be noted that the presence of a wiggler or undulator might have a small effect on the beam dynamics of a light source ring. Since the presence of a wiggler or an undulator only affects the experiment where its synchrotron light from that insertion device is used, the reliability standards of an undualtor or wiggler refrigeration system may not be as critical as it would be for a storage ring bending magnet.

Most light source rings do not have large numbers of devices that operate at cryogenic temperatures below $5 \mathrm{~K}$. The choice of the type of cryogenic refrigeration system is often dictated by the number of low temperature devices in the ring. An example of this is the three Superbend magnets within the Advanced Light Source (ALS) ring at the Lawrence Berkeley National Laboratory. The superconducting bending magnets evenly distributed around a storage ring with a circumference of 196 meters. There are no other devices that use temperature in the range from $4 \mathrm{~K}$ to $10 \mathrm{~K}$. The least expensive cooling system for the ALS Superbend magnets turned out to individual $4 \mathrm{~K}$ cryo-coolers on each of the superconducting magnets [1]. For increased reliability, the Superbend mechanical cryocooler is backed up by an 85-liter bath of liquid helium and a 15-liter bath of liquid nitrogen.

The same type of cryo-cooler that was used on Superbend appears to be an attractive way of cooling other types superconducting insertion devices as well. One can reasonably expect to use one or more cryo-coolers to cool superconducting bending magnets such as the $5 \mathrm{~T}$ bending magnets for the ALS, but cooling long superconducting wigglers and undulators with one or more small cryo-coolers may be more difficult. This section of the report discusses the difference between a wiggler and an undulator and how these differences may affect the performance of the magnet. The effect of the temperature of the magnet beam tube on the gap between the cold magnet poles is discussed. Steps that one might take to reduce the gap between the cold poles are discussed. 


\section{How are Wigglers and Undulators Different?}

The difference between a wiggler and an undulator is subtle. Wigglers and undulators are insertion devices where the field changes polarity as the beam passes along the device. A wiggler or undulator generally, but not always, produces no net bend, thus only has a second order effect on the light source ring beam dynamics. In general an alternating field insertion device is a wiggler when the bend in each half-cell (a section where the field has the same polarity) is much greater than the synchrotron light generation angle $\gamma^{-1}$. The symbol $\gamma$ is defined as the ratio of the accelerated electron mass to the rest mass of an electron. (For the ALS at an electron energy of $1.9 \mathrm{GeV}, \gamma=3718$ ) [2]. An alternating field insertion device is an undulator when the bend per half-cell is much less than the synchrotron-light generation angle $\mathrm{g}^{-1}$. A wiggler or an undulator can be defined in terms of a dimensionless constant $\mathrm{K}$ that represents the ratio of the electron-bending angle to the light generation angle. A definition for $\mathrm{K}$ given in SI units is given as follows [3],[4];

$$
K=\frac{e B_{o} \lambda_{o}}{2 \pi m_{e} c}=93.4 B_{o} \lambda_{o}
$$

where $\mathrm{e}$ is the charge of an electron $\left(1.6022 \times 10^{-19} \mathrm{C}\right) ; \mathrm{B}_{\mathrm{o}}$ is the peak value of the sinusoidal magnetic induction in an insertion device cell; and $\lambda_{u}$ is the cell length (the insertion device period from the peak field point of the field at one polarity to the next peak field point of the same polarity). The value of $\mathrm{m}_{\mathrm{e}}$ is the mass of an electron $\left(\mathrm{m}_{\mathrm{e}}=9.1095 \times 10^{-31} \mathrm{~kg}\right)$; and $\mathrm{c}$ is the velocity of light $\left(\mathrm{c}=2.998 \times 10^{8} \mathrm{~m} \mathrm{~s}^{-1}\right)$. The peak magnetic induction $B_{o}$ is given in $T$; the insertion device period $\lambda_{u}$ is given in meters.

The parameter $\mathrm{K}$ can be regarded as a measure of whether a device is a wiggler or an undulator in the same way that the Knudsen number defines whether a fluid is in continuum flow or free molecular flow. Using the Knudsen number analogy, when $\mathrm{Kn} \gg$, collisions between surfaces dominate fluid flow or heat transfer and collisions between molecules have almost no bearing on a flow or heat transfer process (for example in a vacuum system) [5]. When $\mathrm{Kn} \ll<1$, the flow of a gas or the flow of heat in a gas is entirely dominated by collision between gas molecules. In the region where $\mathrm{Kn}=1$, both free molecular and continuum behavior can be found.

In insertion devices with $\mathrm{K} \gg>1$ definitely defines a wiggler. The radiation from a wiggler comes from different parts of the electron trajectory. This radiation adds incoherently. The highest intensity radiation from a wiggler is similar to that that comes off of a bending magnet (its energy is proportional to machine energy $\mathrm{E}$ squared times the wiggler induction $\mathrm{B}_{\mathrm{o}}$ ). Insertion devices where $\mathrm{K} \ll<1$ are undulators. Radiation from an undulator is quite coherent. The wavelength of the coherent fundamental is well defined and is a function of the device period $\lambda_{u}$ and one over $\gamma^{2}$. There is also a dependence on $\mathrm{K}$. There is no direct dependence on the undulator magnetic induction $\mathrm{B}_{0}$. The coherence that is characteristic of the radiation produced by an undulator extends into the region around $\mathrm{K}=1$ (to $\mathrm{K}=2$ maybe a little higher). As $\mathrm{K}$ moves above 2, the radiation coherence becomes less pronounced, just as free molecular behavior become less pronounced as the Knudsen number drops below one.

From equation 1, it is clear that in order to have an undulator, the magnetic induction must be low or the insertion device period must be small. The undulators that seem to be the most interesting produce intense short wavelength radiation. Not only must the insertion device period be short, but also 
the magnetic induction must be as high as one can make the wavelength short. In order to produce a high magnetic induction in the undulator, one must have a small gap between the poles and strong magnetization in the poles (high currents in the poles) that generates the magnetic field. Since the magnetization of permanent magnet materials is limited, one must try to increase the magnetic field on axis by using superconductors that can operate at very high current densities. Wigglers can have a much larger gap between the poles. Superconductor can be used to increase the magnetic induction on axis. A superconducting undulator might generate a peak induction on axis of about $1 \mathrm{~T}$. A wiggler can generate an induction on axis up to say $8 \mathrm{~T}$ (depending on the superconductor used). With either type of insertion device magnet, having a high current density coil is desirable. For wigglers, an overall coil current density of $1000 \mathrm{~A} \mathrm{~mm}^{-2}$ may be entirely acceptable, but for short period undulators one would like to operate their coils at an overall current density above $1500 \mathrm{~A} \mathrm{~mm}^{-2}$.

Given the state of the art of high temperature superconductors (HTS), it is unlikely that these materials will be used in an undulator anytime soon. HTS coils operate at relatively low current densities, which makes them unsuitable for undulators. The current density in HTS coils is at best borderline as regards to their use in wiggler coils. In both cases, the cost of these conductors is high. The best superconductor candidates for use in either wiggler or undulator coils are niobium titanium and an A-15 material such as niobium tin. This means the magnets must operate at $4 \mathrm{~K}$.

\section{Warm Bore or Cold Bore, the Effect of the Magnet Cryostat on Undulator Performance}

Undulators are characterized by their short periods and as a result their small gaps between their

poles. Superconducting undulators are cryogenic devices that operate at about $4 \mathrm{~K}$. Like all cryogenic devices, it is important to limit the heat that flows into the superconducting magnet and its cryostat. A warm bore magnet will have a lower heat leak into the cryostat than will a cold bore magnet. Cryogenic refrigeration (particularly when one uses small cryo-coolers) dictates that the magnet bore should be at room temperature. We shall see later that cryo-cooler cooling is possible for undulators that have beam bore tubes at $30 \mathrm{~K}$ or above. Undulator performance in the most extreme cases suggests that the magnet should have a cold bore at or near $4 \mathrm{~K}$. This section will look at the effect of the bore temperature on the magnetic performance of superconducting wigglers and undulators.

In order to demonstrate the effect of the cryostat on the field generated by an insertion device, an air core coil device was calculated. Field is generated using superconducting coils that operate at a current density j. There is no pole. The space that would normally be occupied by a pole piece is filled with the superconducting coil, so the width of an individual coil is set at half the magnet period $\lambda$. The magnetic induction $\mathrm{B}_{\mathrm{o}}$ is calculated at the center of the gap between the coils. The free gap for the magnet $\mathrm{G}$ was fixed at $5 \mathrm{~mm}$ and $8 \mathrm{~mm}$ for the calculations. The coil thickness $\mathrm{t}$ and the coil overall current density $\mathrm{j}$ was the same for all the cases studied. For the calculations, the insertion device period $\lambda$ was changed and the thickness of the cryostat around the pole $y$ was changed. Figure 1 shows the magnet cross-section for the region around the gap for a pure superconducting insertion device with no pole pieces. The cross-section is taken along the direction of the beam at the magnet center. Figures 2 and 3 show the calculated value for the peak on axis induction $B_{o}$ as a function of the device period $\lambda$ and the cryostat thickness y for free magnet gaps of $5 \mathrm{~mm}$ and $8 \mathrm{~mm}$ respectively. 


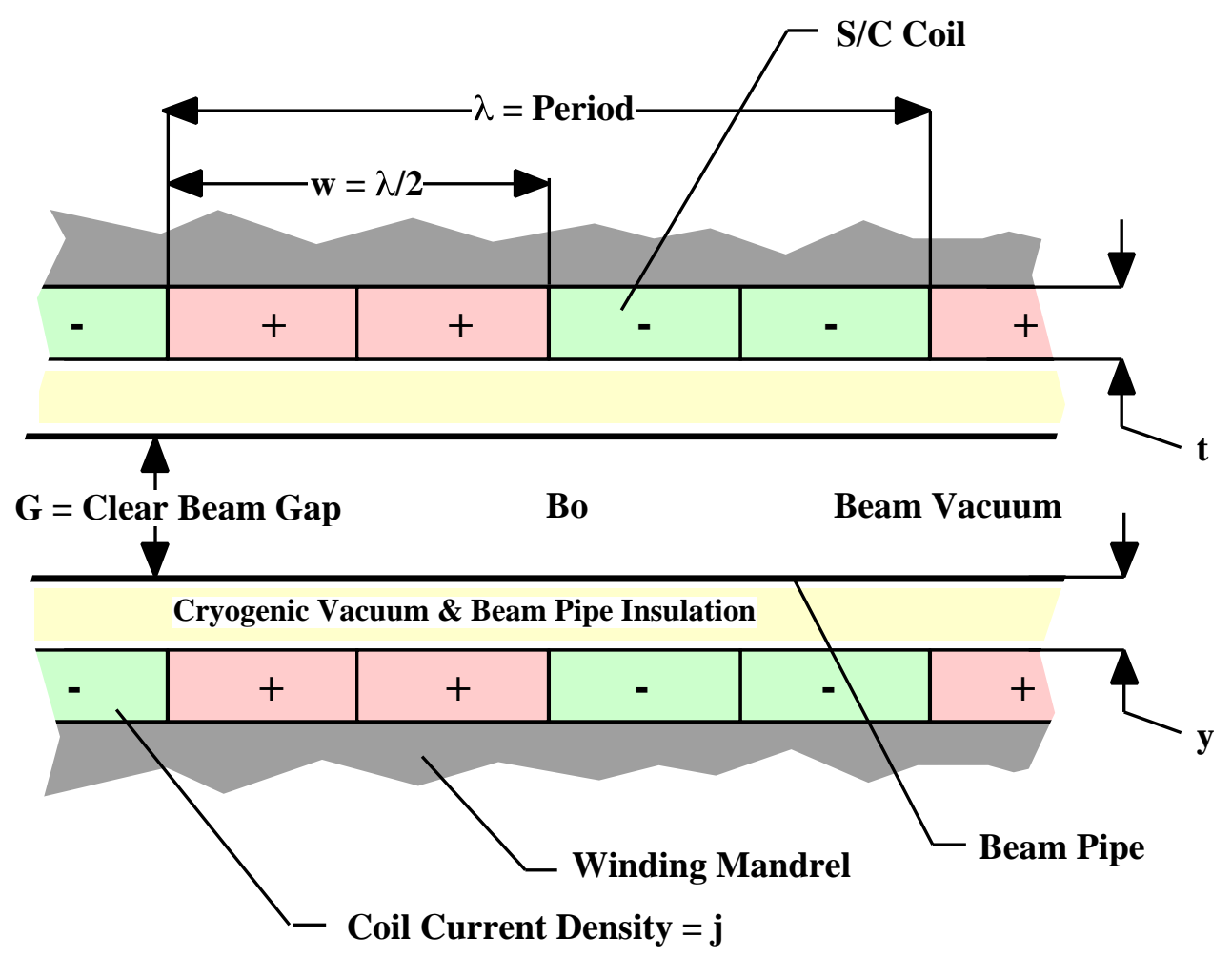

Figure 1. A Cross-section of a Pure Superconducting Insertion Device along the Beam Axis in the Region of the Magnet Gap

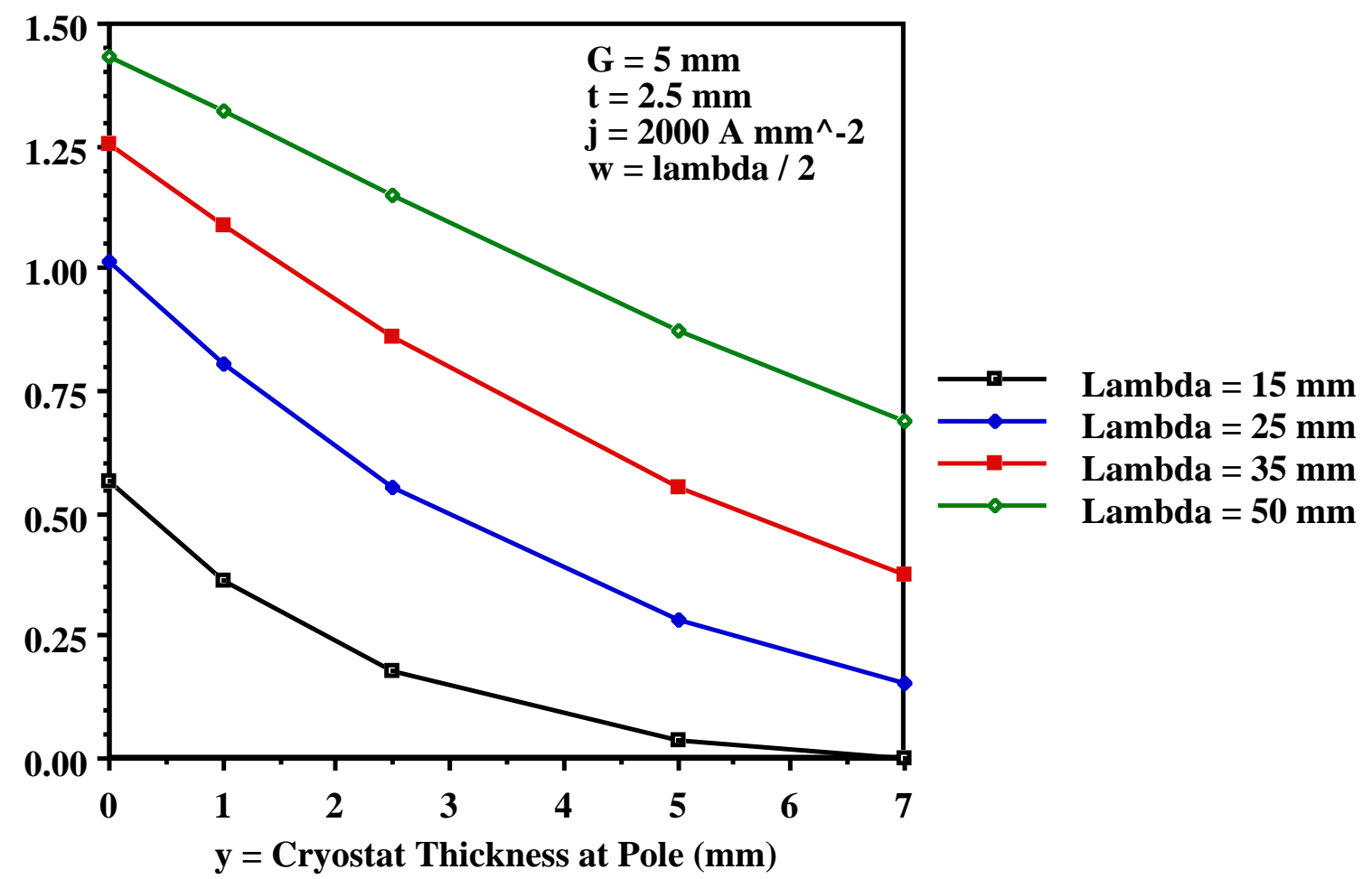

Figure 2. The Peak On-axis Magnetic Induction Amplitude Bo at the Center of a 5-mm Free Gap Eight Cell Insertion Device As a Function of the Device period $\lambda$ and the Cryostat thickness in the Bore Region y 


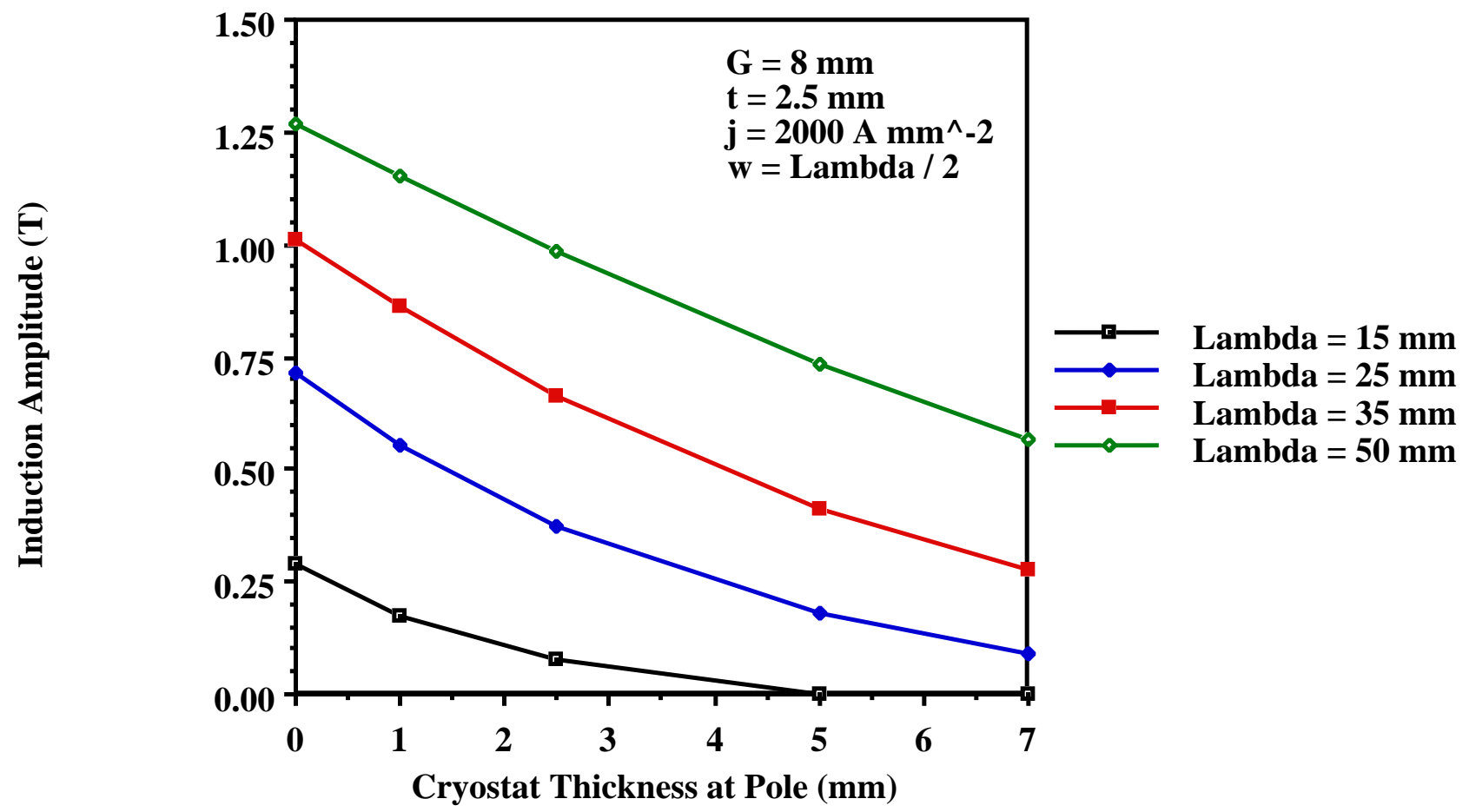

Figure 3. The Peak On-axis Magnetic Induction Amplitude Bo at the Center of a 8-mm Free Gap Eight Cell Insertion Device As a Function of the Device period $\lambda$ and the Cryostat thickness in the Bore Region y

Figures 2 and 3 show the kind of behavior that one might expect. The long period devices generated a higher peak field on axis than the short period devices. This is true for both free gaps and at all values of cryostat thickness in the bore region. The magnet with the 8-mm free gap had a lower field than the magnet with the smaller free gap. The number of ampere-turns around the pole is the same for both cases at a particular period. For both cases, the coil current density $\mathrm{j}=2000 \mathrm{~A} \mathrm{~mm}^{-2}$. Increasing $\mathrm{j}$ will increase the peak on axis field. At both free gaps, the device with a period of $50 \mathrm{~mm}$ always will behave like a wiggler. The short period device with a period of $15 \mathrm{~mm}$ will behave like an undulator. What is clear from all of the cases studied, the peak on axis induction always goes down as the free gap is increased and it always goes down as the cryostat thickness y is increased.

The peak on axis induction values shown for the eight-cell devices shown in Figures 2 and 3 will be higher if the device has a ferromagnetic pole (say soft iron or holmium) to concentrate the magnetic flux. The configuration shown in Figure 1 is not optimum for a device that has ferromagnetic poles. The effect of the ferromagnetic material is to reduce the number of ampere-turns needed to produce a given level of magnetic flux on axis. When the ferromagnetic poles saturate, the $\Delta \mathrm{i}$ needed to produce a given $\Delta \mathrm{B}$ will decrease to a value commensurate with an air core device. However, the magnetization present in the ferromagnetic material always is added to the magnetization created by the currents around the poles. To do these calculations correctly, a three-dimensional code that uses materials with finite and variable permeability is needed to do the field calculations. 


\section{Steps to Reduce the Thickness of the Cryostat in the Bore Region}

Figures 2 and 3 illustrate the effect of the cryostat thickness in the region near the bore on the peak on axis field generated by the coil current in the insertion device. It is clear that reducing the cryostat thickness to a minimum value is desirable regardless of the details of the coil and pole design. The elements that make up the cryostat in the bore region of a superconducting wiggler or undulator consist of one or more of the following; 1) the wiggler or undulator beam vacuum tube, 2) two or more layers of multi-layer insulation (MLI), 3) an actively cooled shield at say $50 \mathrm{~K}$, and 4 the inner wall of a helium cryostat vessel that contains the superconducting coils.

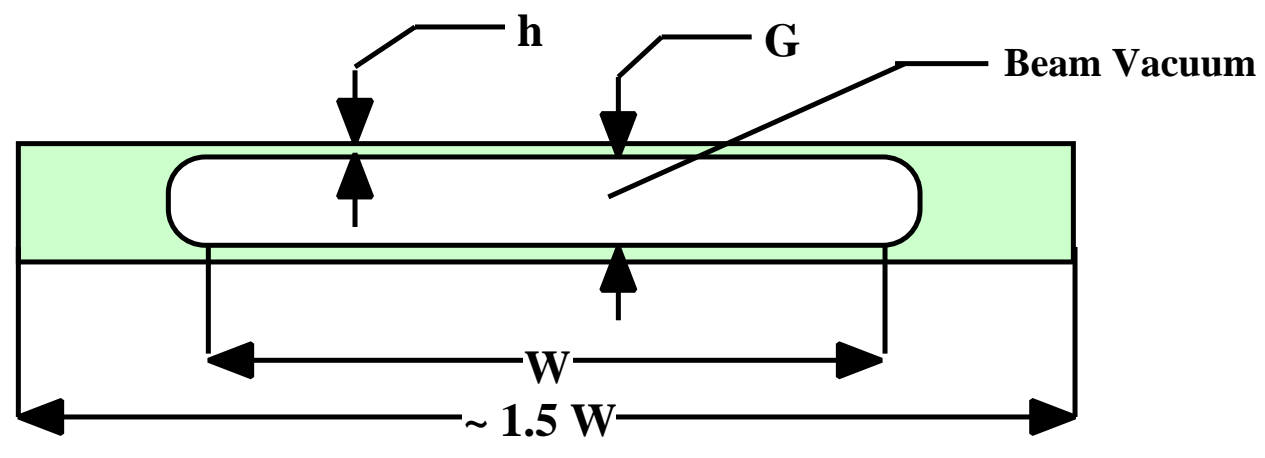

Figure 4. A Typical Configuration for the Beam Vacuum Tube Used in a Superconducting Insertion Magnet

The beam vacuum tube for an insertion magnet must be designed to be under vacuum even if the magnet cryostat vacuum is up to air. Figure 4 shows a typical configuration that one might use for an insertion magnet beam tube. The minimum thickness of the beam pipe $\mathrm{h}$ is dictated by its width $\mathrm{W}$ and the design stress $\sigma_{\mathrm{d}}$ of the material that beam tube is fabricated from. In general, the vacuum tube should be designed to operate at $2 / 3$ of the material yield stress, or $1 / 3$ of the material ultimate stress, whichever is more conservative. An approximate expression for the minimum beam tube thickness can be derived from simple beam theory. This expression is given as follows;

$$
h=\left[\frac{P}{2 \sigma_{d}}\right]^{0.5} W
$$

where $\mathrm{P}$ is the design pressure on the inside or the outside of the beam vacuum pipe $\left(\mathrm{P}=1.013 \times 10^{5} \mathrm{~Pa}\right)$. For most bore tube materials the design stress $\sigma_{\mathrm{d}}$ is about a factor of 300 to1500 higher than P, so the thickness of the bore tube $\mathrm{h}$ is from 0.02 to 0.04 times the dimension $\mathrm{W}$. One can reduce the bore tube wall thickness $h$ by reducing the effective value of $\mathrm{W}$. This can be done by making the vacuum crosssection an ellipse with a minor dimension $\mathrm{G}$ and the major dimension $\mathrm{W}$. It will be hard to build a bore tube out of a reasonable material that has a thickness that is less than $0.5 \mathrm{~mm}$. For half hard aluminum the bore tube thickness is from 0.8 to $1.2 \mathrm{~mm}$ for a $\mathrm{W}=40 \mathrm{~mm}$. For a half hard copper bore tube with a width $\mathrm{W}=40 \mathrm{~mm}$, the minimum bore tube thickness is 0.85 to $1 \mathrm{~mm}$. A 304 stainless steel bore tube about $0.7 \mathrm{~mm}$ thick $(\mathrm{W}=40 \mathrm{~mm})$ can be fabricated, but it must have a copper coating on the inside. 
Multi-layer insulation (MLI) serves two functions [5]. It reduces the radiation transmission from the warm wall to the cold wall by reducing the effective emissivity of the radiating surfaces. MLI can also reduce the transmission of heat by free molecular conduction when the cryostat vacuum is bad. The radiation heat transfer and the free molecular heat conduction through MLI is inversely proportional to the number of layers of insulation. Since it is desirable to make the insulation stack of minimum thickness (maximum density) in the bore region of an insertion device, the MLI should also have a separator layer between sheets. A good separator material is sheer open weave polyester bridal veil netting. The netting improves the insulation performance when the insulation is compressed. When one uses netting between layers the heat leak through the MLI stack goes as the square root of the pressure on the insulation stack [6]. For this reason, a combination of MLI and bridal veil netting is the insulation of choice in flexible transfer lines where local pressures (over a small area) across the insulation can be quite high. The MLI on other parts of the insertion device cryostat can be loose with many layers. Fortunately the region around the insertion device bore tube represents a relatively small area compared to the rest of the cryostat.

If one wants to reduce the thickness of the cryostat at the magnet bore, one can eliminate the intermediate temperature shield between the magnet that is at $4 \mathrm{~K}$ and the beam vacuum tube. Since the beam vacuum tube dimension scales with the useful gap $\mathrm{G}$, eliminating the intermediate temperature shield probably makes sense when $\mathrm{G}$ is small (a typical undulator), but it may not make sense when $\mathrm{G}$ is large (a typical wiggler). If an intermediate temperature shield is in the insulation around the beam tube, it can be expected to add between 0.5 and $1.0 \mathrm{~mm}$ to the thickness of the cryostat in this region. A beam vacuum tube that operates at below $100 \mathrm{~K}$ does not need an intermediate temperature shield between it and the magnet. An intermediate shield around the beam vacuum tube is only a consideration when the beam tube is at room temperature. In a small gap undulator, with $\mathrm{G}<7 \mathrm{~mm}$, it is likely that there would be no shield around the beam tube even if it operates at room temperature.

Whether or not there is a helium vessel around the magnet, depends on whether the magnet is cooled in a bath of liquid helium or cooled indirectly by conduction to a cryo-cooler or a two-phase helium flow circuit. If the insertion magnet is cooled by conduction, there is no need for a helium vessel. If the insertion magnet has a cold bore at $4 \mathrm{~K}$, it is probable that the magnet will have to be cooled in a helium bath. The extra heat into the magnet from the cold bore will probably make conduction cooling of the magnet problematical, particularly if the magnet is fabricated with niobium titanium. The heat coming into a $4 \mathrm{~K}$ bore tube may be quite large compared to all of the other $4 \mathrm{~K}$ heat loads. Heat going into the beam tube (at any temperature) will be heat coming into the ends of the beam tube by radiation and conduction plus any heat that is deposited into the beam tube due to beam heating. The issue of beam heating will be discussed later in this report.

It is highly unlikely that a $4 \mathrm{~K}$ bore insertion magnet can be cooled using one or more small cryocoolers. As a result, the refrigerator needed to cool the insertion magnet will be large enough to produce the liquid helium needed to cool the magnet coils, the leads, and the cold bore tube. When the insertion magnet has a $4 \mathrm{~K}$ bore, the beam vacuum chamber must be part of the helium vessel for the magnet cryostat. Since a helium bath cryostat should be designed for pressures of 0.2 to $0.3 \mathrm{MPa}$, the thickness of the beam tube vacuum chamber will have increase by a factor of 1.4 to 1.7 over the beam tube thickness needed to hold off $0.1 \mathrm{MPa}$ (1 bar). 
Table 1. The Thickness of the Insertion Device Cryostat at the Beam Vacuum Chamber $\mathbf{y}$

As a Function of Beam Tube Temperature and Beam Vacuum Chamber Gap G

Beam Tube T

$300 \mathrm{~K}$

30 to $80 \mathrm{~K}$

$4.4 \mathrm{~K}$

$$
\begin{aligned}
& \text { Cryostat Thickness a } \\
& \mathrm{mm}=10 \mathrm{~mm}
\end{aligned}
$$

5.4

2.6

1.3
6.3

3.5

2.6
Beam Tube $\mathbf{y}(\mathrm{mm})$

$\mathrm{G}=15 \mathrm{~mm} \quad \mathrm{G}=20 \mathrm{~mm}$

7.2

8.1

4.4

5.3

3.9

The following is assumed in the table above. The beam tube width $\mathrm{W}=8 \mathrm{G}$. The beam vacuum tube is made from 6061 aluminum with a design stress of $104 \mathrm{MPa}$. Ten layers of MLI are used for the $300 \mathrm{~K}$ bore case; three layers of MLI are used for the 30 to $80 \mathrm{~K}$ bore case. There is no MLI at $4.4 \mathrm{~K}$. Each MLI layer is $0.4 \mathrm{~mm}$ thick. An additional space of $0.5 \mathrm{~mm}$ is added for vacuum pumping. At $4.4 \mathrm{~K}$, the beam tube forms part of the cryostat helium vessel.

Table 1 shows the calculated thickness of the insertion magnet cryostat around the beam vacuum $y$, for various values of the bore temperature and free gap $\mathrm{G}$ within the beam vacuum tube. It is clear from Table 1 that there is an incentive to reduce the beam bore temperature for insertion devices that have a small beam gap. As the insertion device gap $G$ increases, there is less incentive to reduce the gap temperature. The calculated values for y are based on using a 6061 bore tube that has been heat-treated back to the T6 state. There appears to be little incentive to go to 304 stainless steel or Inconel in the bore tube in order to reduce its thickness. Even in the smallest gap case, a change in the beam tube material will make less than a 25 percent change in the value of $y$. Larger gains in reducing the bore tube thickness $h$ can be made by reducing its width $\mathrm{W}$. In many cases the aspect ratio of the inside of the beam tube $\mathrm{W}$ over $\mathrm{G}$ is chosen in an arbitrary way. From the standpoint of reducing the cryostat thickness around the beam vacuum, the beam tube aspect ratio should be no larger than necessary.

When one looks at the $\mathrm{G}=20 \mathrm{~mm}$ case, one sees that there is virtually no incentive to reduce the beam tube temperature from the 30 to $80 \mathrm{~K}$ range to $4.4 \mathrm{~K}$. Because the cost of the refrigerator for cooling a $4.4 \mathrm{~K}$ gap magnet is much higher, there is a strong incentive not to have a $4.4 \mathrm{~K}$ bore on a large gap device. It appears that in all of the cases shown above that reducing the bore tube temperature to the $30 \mathrm{~K}$ to $80 \mathrm{~K}$ range may make sense. It can also be argued that using a superconductor that will increase the average current density in the coil (niobium tin designed for low field use) will permit one to build a small gap insertion device with a room temperature bore instead of a low temperature bore.

The physical size of the insertion device in cross-section approximately scales with the gap between the magnet poles $G_{M}$. See Figure 5 for a cross-section view. The value of $G_{M}$ is given as follows:

$$
G_{M}=G+2 y
$$

The mass of the insertion device, hence the heat leak into the cryostat through its cold mass supports will scale with the device length and $\mathrm{G}_{\mathrm{M}}$ squared. The heat leak into the cryostat by conduction and radiation through the cryostat insulation on the outside of the device is proportional to the surface area of the device, which also scales with $\mathrm{G}_{\mathrm{M}}$ to some power less than 2 . It should be noted that the cost of the insertion device itself scales with $\mathrm{G}_{\mathrm{M}}$ to about the 1.4 power [7],[8]. 


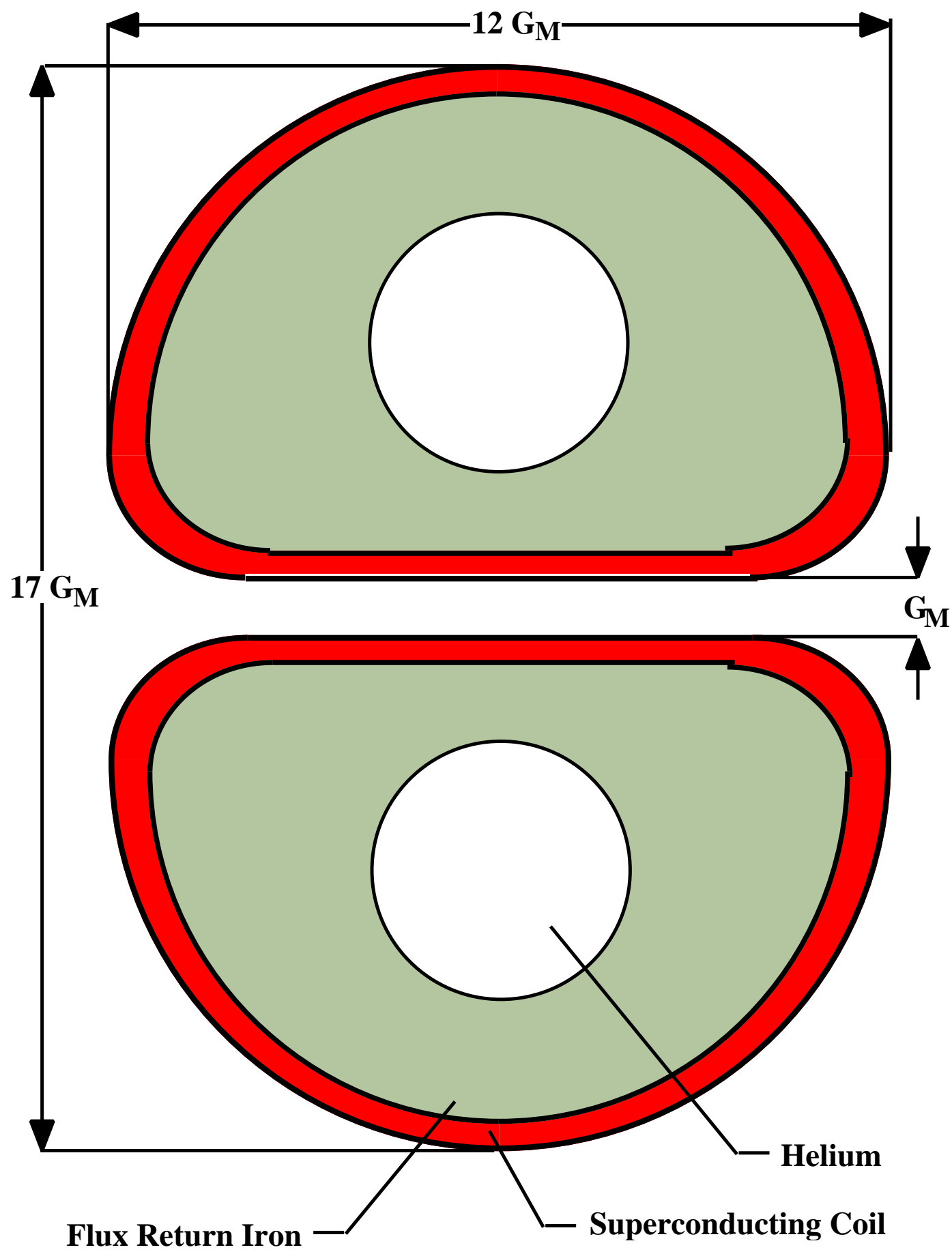

Figure 5. A Cross-section View of an Insertion Device Coil Package showing the Height and Width of the Coil Package in Units of the Physical Gap $\mathrm{G}_{\mathrm{M}}$ between the Superconducting Coils (Note: the width of the insertion device cryostat is 80 to $100 \mathrm{~mm}$ wider than the coil package shown above.) When $\mathrm{G}_{\mathrm{M}}=15 \mathrm{~mm}$, the total cryostat width can be expected to from 260 to about $300 \mathrm{~mm}$. The cryostat height will be at least $400 \mathrm{~mm}$, when $\mathrm{G}_{\mathrm{M}}=15 \mathrm{~mm}$. (When $\mathrm{G}_{\mathrm{M}}=15 \mathrm{~mm}, \mathrm{G}=8.2 \mathrm{~mm}$.) 


\section{HEAT FLOW INTO THE INSERTION DEVICE}

\section{Heat Transfer to a Cold Insertion Device Vacuum Chamber}

The use of a cold bore tube in an insertion magnet means that additional heat is transferred into the cold region of the magnet cryostat. This additional heat comes from five sources; 1) conduction from $300 \mathrm{~K}$ along the beam tube, 2) thermal radiation down the beam tube from the room temperature ends, 3 ) heating due to a loss of electrons from the beam, 4) synchrotron radiation heating from radiation sources up stream from the insertion device and 5) heating due to image currents that are induced in the beam tube from the storage ring beam. A room temperature beam tube has heating from stray electron beam, synchrotron radiation heating, and the image current heating. In general, these sources of heat in an insertion device with a room temperature beam tube can be removed by water-cooling.

Heating due to thermal conduction from $300 \mathrm{~K}$ into an insertion device bore tube is proportional to the circumference of the bore tube and its thickness $\mathrm{h}$. The heat leak into a bore tube $\mathrm{Q}_{\mathrm{c}}$ from $300 \mathrm{~K}$ to the bore tube temperature $\mathrm{T}_{1}$ can be estimated from the following expression;

$$
Q_{c}=\frac{2 \Gamma A_{c}}{L_{c}}=\frac{2 \Gamma(2 W+\pi G) h}{L_{c}}=2 \Gamma(2 W+\pi G) \frac{W}{L_{c}}\left[\frac{P}{\sigma_{d}}\right]^{0.5}
$$

where $\mathrm{A}=$ the cross-section area of the tube; $\mathrm{G}$ is the bore free gap; $\mathrm{W}$ is the width of the bare tube (see Figure 4); and $\mathrm{L}_{\mathrm{c}}$ is the length of the low conductivity section of the tube. $\mathrm{P}$ is the design pressure for the tube section $\left(\mathrm{P}=1.013 \times 10^{5} \mathrm{~Pa}\right)$ and $\sigma_{\mathrm{d}}$ is the design stress for the material that is in the low heat leak section of the bore tube. ( $\sigma_{\mathrm{d}}$ is typically one third of the ultimate stress for the bore tube material.). $\Gamma$ is the thermal conductivity integral for the material that makes up the low heat leak section of the bore tube. $\Gamma$ is defined as follows;

$$
\Gamma=\int_{\mathrm{T} 1}^{\mathrm{T} 2} \mathrm{k}(\mathrm{T}) d T
$$

where $\mathrm{k}(\mathrm{T})$ is the thermal conductivity of the low heat leak bore tube material as a function of temperature $\mathrm{T}$. $\mathrm{T} 2$ is room temperature $(\mathrm{T} 2=300 \mathrm{~K})$ and $\mathrm{T} 1$ is the temperature of the magnet gap. For a typical undulator magnet with a free gap $\mathrm{G}=8 \mathrm{~mm}$, and $\mathrm{W}=40 \mathrm{~mm}, \mathrm{~h}=0.7 \mathrm{~mm}$ and $\mathrm{L}_{\mathrm{c}}=150 \mathrm{~mm}$. If the low heat leak bore tube section is made from 304 stainless steel with a flash of 2 microns of copper $\Gamma=3100 \mathrm{~W} \mathrm{~m}^{-1}$, then $\mathrm{Q}_{\mathrm{c}}=3.04 \mathrm{~W}$. If the same bore tube is made from 5456 aluminum, the heat leak $\mathrm{Q}_{\mathrm{c}}$ would be about ten times as large as for 304 stainless steel, unless the transition section is made longer. The heat leak for either material may be acceptable provided the magnet bore tube temperature is above 30 to $50 \mathrm{~K}$. If the magnet bore tube is at $4.2 \mathrm{~K}$ or lower, a two-stage transition section will needed. There must be an intermediate temperature point with a temperature of say $40 \mathrm{~K}$ that is half way between the $300 \mathrm{~K}$ section and the $4 \mathrm{~K}$ section in terms of distance. If the intermediate temperature is about $40 \mathrm{~K}$ and the overall length of the transition is $150 \mathrm{~mm}$, the heat leak into the $4 \mathrm{~K}$ region would be about $0.1 \mathrm{~W}$ for a stainless steel transition tube and about 1 watt for an aluminum transition tube section. In general, the lower the temperature of the magnet bore, the longer the transition section should be. Figure 6 illustrates a single-stage and a two-stage bore tube end transition for $\mathrm{G}=8 \mathrm{~mm}$. 


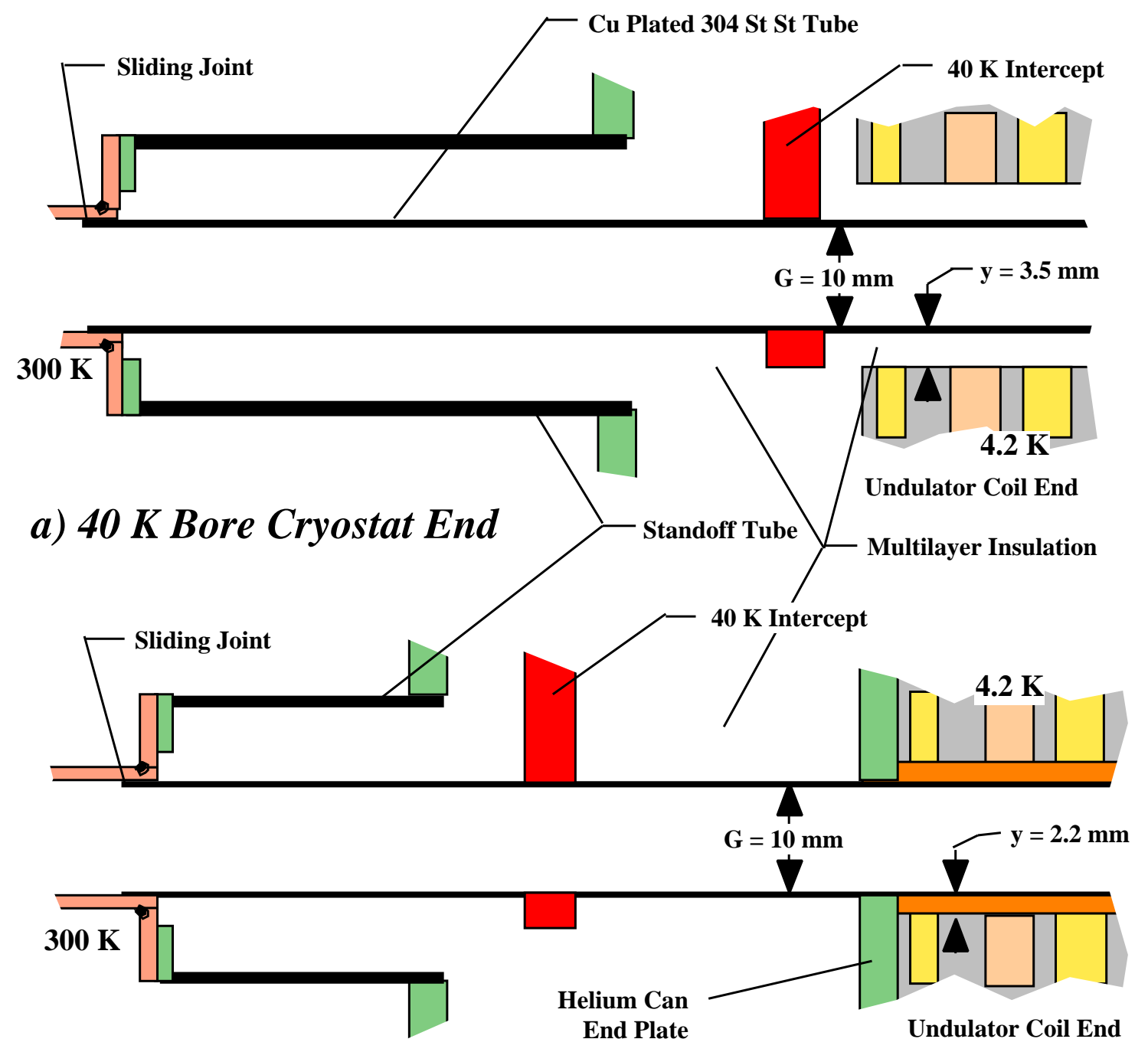

b) 4.2 K Bore Cryostat End

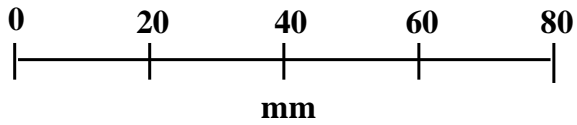

Figure 6. End Transitions for Insertion Device Cryostats with a $40 \mathrm{~K}$ Bore and a $4.2 \mathrm{~K}$ Bore with a $40 \mathrm{~K}$ intercept

Heat leak into the cold bore by radiation is proportional to the opening area of the bore tube entering the magnet. An approximate expression for the radiation heat leak into the cold bore of an insertion magnet is as follows;

$$
Q_{R}=2 \sigma\left(W G+\frac{\pi G^{2}}{4}\right)\left[T_{2}^{4}-T_{1}^{4}\right]
$$

where G, W, $T_{2}$ and $T_{1}$ have been previously defined. $\sigma$ is the Stefan Bolzmann constant $\left(\sigma=5.67 \times 10^{-8}\right.$ $\mathrm{W} \mathrm{m}{ }^{-2} \mathrm{~K}^{-4}$ ). Equation 6 assumes a form factor of 1 and an emissivity of 1 , because radiation into a hole 
behaves like radiation into a black body with the cross-section area of the hole (in this case two holes). For the case where $\mathrm{G}=8 \mathrm{~mm}, \mathrm{~W}=40 \mathrm{~mm}$, the radiation heat load into the bore $\mathrm{Q}=0.34 \mathrm{~W}$. If the bore tube is at $4 \mathrm{~K}$, the radiation heat leak into the $4 \mathrm{~K}$ region will be at least a factor of three lower, because of the thermal staging that is needed to reduce the heat leak due to conduction down the bore transition section. The radiation heating per unit inner bore tube area will be highest near the ends. The radiation heating per unit area will fall off rapidly as one proceeds deeper along the magnet bore tube.

\section{Heating Due to Synchrotron Radiation and Beam Electrons}

The heating due to electrons from the beam and synchrotron radiation from sources upstream from the insertion device is a function of the details of the ring design and the degree to which the insertion device bore tube is shielded from these sources. Electron beam heating will be highest where a beam blow up occurs. One should understand when and where beam blow occurs in the ring, in order to control this source of heating to the insertion device. Synchrotron radiation can penetrate the bore tube, if the energy of this radiation is high. This may be of some concern, because it may add to the heat that is put into the superconducting coils. Most of the synchrotron radiation heating will occur in the bore tube itself. If the bore tube is at $300 \mathrm{~K}$, this is of little concern, because the heat can be removed by cooling the bore tube. Synchrotron radiation heating in a low temperature bore tube is of concern, because this heat must be removed by the cryogenic refrigerator. In general, the lower the bore tube temperature, the more synchrotron radiation heating is of concern. Heating from beam electrons comes from multiple scattering within the bore tube. This can also contribute an extra heat load to the superconductor. In general, if heating from the beam is large enough to be of concern for superconductor operation, considerable radiation damage is being done to the magnet insulation. A large amount of beam heating suggests that there are large beam losses in the machine. Electron beam heating in a low temperature bore tube is of concern, because this heat must be removed by the cryogenic refrigerator. In general, the lower the bore tube temperature, the more electron beam heating is of concern. Magnet bore heating due to synchrotron radiation and stray electrons from the beam is difficult to quantify. In general, one wants to do all one can to eliminate these source of bore tube heating when the insertion device bore tube is at cryogenic temperature.

\section{Beam Heating in a Cold Insertion Device Vacuum Chamber}

Heating due to image currents from the beam may be the largest single source of heat into a cold insertion device bore tube. Image current heating first became important when the first cold bore superconducting dipole and quadrupoles were being considered for accelerators. One of the first such machines was the experimental superconducting storage ESCAR at Berkeley in the mid 1970's. Beam heating in ESCAR was of concern because of the extra heating that would be applied to the cold bore of the ESCAR magnets. Beam heating had to be added to the static and AC loss $4 \mathrm{~K}$ heating from the rest of the machine. ESCAR was to be a proton storage ring with proton beams that were not quite fully relativistic (where $\beta$ was between 0.8 and 0.95). D. B. Hopkin's of the Lawrence Berkeley Laboratory developed a beam heating equation in the early 1970's, which took the skin effect into consideration $[9,10]$. The anomalous skin effect was not a part of this equation because the equation had been applied 
to cases with low beam frequency (less than $100 \mathrm{MHz}$ ). The Hopkin's equation for beam heat per unit length (P/l) took the following general form:

$$
\frac{P}{\ell}=0.027 \frac{N^{2} \beta^{5 /} c^{5 / 2} \alpha^{2} \mu^{1 / 2} \rho_{v}^{1 / 2}}{C_{M} R m L_{B}^{3 / 2}}
$$

where $\mathrm{N}$ is the number of particles in the machine; $\beta=\mathrm{v} / \mathrm{c}$ (for electrons in a light source $\beta=1$; $\mathrm{c}$ is the velocity of light $\left(\mathrm{c}=2.997 \times 10^{8} \mathrm{~m} \mathrm{~s}^{-1}\right)$; $\alpha$ is the particle charge (for electrons $\alpha=1.592 \times 10^{-19} \mathrm{C}$ ); $\mu$ is the permeability of the tube wall (for 304 stainless-steel and nonferrous materials $\mu=\mu_{\mathrm{o}}=4 \pi \times 10^{-7} \mathrm{H} \mathrm{m}^{-1}$ ); and $\rho_{v}$ is the volume resistivity for the wall material. $C_{M}$ is the circumference of the machine; $R$ is the distance from the beam center to the resistive wall; $m$ is the number of bunches in the machine and $L_{B}$ is the bunch length.

If one defines the machine beam current for with the following expression (with $\beta=1$ for electrons in a storage ring);

$$
I=\frac{N \alpha c}{C_{M}}
$$

$-7 b-$

The equation for heating per unit length will take the following form that can be directly compared with later versions of the beam heating equation;

$$
\frac{P}{\ell}=0.027 \frac{I^{2} c^{1 / 2} \mu^{1 / 2} \rho_{v}^{1 /} \mathcal{E}_{M}}{R m L_{B}^{3 / 2}}
$$

Equation 7c uses the standard definition of skin depth, which applies when the resistivity of the wall material is high and when the frequency of the RF generated by the passing beam is low. The beam heating per unit length is proportional to the beam current squared, and the machine circumference. The beam heating per unit length is inversely proportional to the number of bunches. In other words if all of the beam current is contained in a single bunch, the beam heating is higher. The longer the bunch length, the lower the beam heating per unit length. Beam heating per unit length is inversely proportional to the radius of the beam pipe (the distance between the center of the beam and the pipe wall). A beam pipe that is two parallel plates has half the beam heating of a circular bore tube, so the shape of the bore is of limited usefulness in controlling beam heating. The largest controllable factor on beam heating per unit length for a given machine is the resistivity of the beam tube wall surface.

Because of the skin effect the amount of low resistivity material needed to achieve low beam heating is quite small. An expression for the image current thickness $\delta$ can be given as follows;

$$
\delta=\left[\frac{2 \rho_{v}}{\omega \mu}\right]^{0.5}=\left[\frac{\rho_{v} L_{B}}{\pi c \mu}\right]^{0.5}
$$

The value of $\delta$ given by Equation 8 represents the thickness for the lowest frequency $f=\omega / 2 \pi$ image current components of RF from the passing beam. The surface resistance of the bore tube material is; 


$$
R_{S}=\left[\frac{\omega \mu \rho}{2}\right]^{0.5}=\left[\frac{\pi c \mu \rho}{L_{B}}\right]^{0.5}
$$

The anomalous skin effect was not considered in the derivation of Equations 7a, 7b, 7c, 8, or 9. Note, the second term comes from Hopkins [10], the first term comes from Chu and Reggiero [11].

The anomalous skin effect theory predicts the following surface resistance (based on an interpolation formula for the so-called diffusion model [12]

$$
R_{s}=R_{\infty}\left(1+1.157 \alpha^{-0.276}\right) \text {, for } \alpha \geq 3
$$

where the dimensionless parameter $\alpha$ is given by;

$$
\alpha=\frac{3}{4} \omega \mu(\rho \lambda)^{2} \rho^{-3}
$$

and $R_{\infty}$ is a quantity independent of temperature and impurity, having a $\mathrm{f}^{2 / 3}$ dependence on frequency;

$$
R_{\infty}=\left(\frac{3^{1 / 2}}{16 \pi}(\rho \lambda)(\omega \mu)^{2}\right)
$$

For a given metal the product of metal volume resistivity $\rho$ and the metal electron mean free path for the metal $\lambda$ is a constant. For copper $\rho \lambda=6.52 \times 10^{-16} \Omega \mathrm{m}^{2}$. Metals such as aluminum, gold, and silver have similar values for $\rho \lambda$. See Table 2 below for values of the $\rho \lambda$ product for metals. For pure metals, the resistivity changes rapidly as one goes down in temperature. Thus when uses a pure copper beam pipe $\alpha$ will be small at room temperature and large at low temperature. The resistivity of pure copper, silver, gold and aluminum at $300 \mathrm{~K}, 40 \mathrm{~K}$ and $4.2 \mathrm{~K}$ are also shown in Table 2 . In addition, aluminum has a low magneto-resistivity compared to copper, silver, or gold.

Table 2. The value of the $\rho \lambda$ Product and $\rho$ at Various Temperatures for Very Pure Metals that might be used for Undulator Magnet Beam Tubes

$\begin{array}{ccccc}\text { Metal } & \rho \lambda & \rho \text { at } 300 \mathrm{~K} & \rho \text { at } 40 \mathrm{~K} & \rho \text { at } 4.2 \mathrm{~K} \\ & \left(\Omega \mathrm{m}^{2}\right) & (\Omega \mathrm{m}) & (\Omega \mathrm{m}) & (\Omega \mathrm{m}) \\ \text { Copper } & 6.52 \times 10^{-16} & 1.74 \times 10^{-8} & 2.95 \times 10^{-10} & 5.53 \times 10^{-11} \\ \text { Silver } & 8.43 \times 10^{-16} & 1.59 \times 10^{-8} & 2.9 \times 10^{-10} & 1.62 \times 10^{-10} \\ \text { Gold } & 8.35 \times 10^{-16} & 2.46 \times 10^{-8} & 4.1 \times 10^{-10} & 2.5 \times 10^{-10} \\ \text { Aluminum } & 3.97 \times 10^{-16} & 2.68 \times 10^{-8} & 2.1 \times 10^{-10} & 9.0 \times 10^{-12}\end{array}$

The value of RRR for copper is 300; the RRR for silver and gold are about 100; and the RRR for aluminum is 3000 . 
For a copper bore tube or a stainless steel bore tube lined with a layer of copper, at $300 \mathrm{~K}$ the surface resistance is calculated using equation 9. At low temperatures below say $80 \mathrm{~K}$, one might use Equation 10 to calculate the surface resistance. For pure copper at say $20 \mathrm{~K}$ the surface resistance is $\mathrm{R}_{\infty}$ as calculated using Equation 12. If the beam tube is at an intermediate temperature say 40 to $80 \mathrm{~K}$ and the metal on the inner wall is not pure so that $\rho \geq 3 \times 10^{-9} \Omega \mathrm{m}$, the anomalous skin effect will not be an important factor in determining the amount of heat produced on the beam tube surface.

The beam heating per unit length from the Chu and Reggiero paper takes the following general form once the integration of product of bunch spectrum squared $[\lambda(\omega)]^{2}$ surface resistance $R_{S}(\omega)$ with respect to $\omega$ has occurred;

$$
\frac{P}{\ell}=\Gamma\left(\frac{3}{4}\right) \frac{2^{1 / 2} C_{M} \mu^{1 /} \rho^{1 / 2} c^{1 / 2}}{8 \pi^{2}} \frac{I^{2}}{m L_{B}^{3 / 2}}
$$

where $\Gamma(3 / 4)$ in the gamma function of $3 / 4$ and all the other terms are defined the same as they are in equation 7c. An alternative version of the this equation uses the a basic impedance term $\mathrm{z}_{0}$, where this term is defined as follows;

$$
z_{o}=c \mu_{0}=120 \pi=376.6 \Omega
$$

The modified equation that uses the baseline impedance term takes the following form;

$$
\frac{P}{\ell}=\Gamma\left(\frac{3}{4}\right) \frac{2^{1 / 2} C_{M} z_{o}^{1 / 2} \rho^{1 / 2}}{8 \pi^{2}} \frac{I^{2}}{m L_{B}^{3 / 2}}
$$

Since $\Gamma(3 / 4)=1.2254$, the constant term of Equations 13 and $14 \mathrm{~b}=0.02195$, which is less than the constant term in Equation 7c, which is about 0.027. It is not clear why there is a difference between Equations 7c and 13.

When the RF frequencies are high and the wall material resistivity is low, the anomalous skin effect theory applies $(\alpha>>10)$. Equation 12 can be used to describe the surface resistance. In extreme cases where a is large (say $\alpha>500$ ), one can use the following expression to estimate the beam heating per unit length along the bore tube [13];

$$
\frac{P}{\ell}=\Gamma\left(\frac{5}{6}\right) \frac{3^{1 / 6}}{8 \pi^{2}(2 \pi)^{1 / 3}} \frac{C_{M} z_{o}^{2 / 3}(\rho \lambda)^{1 / 3}}{R L_{B}^{5 / 3}} \frac{I^{2}}{m}
$$

A second form of Equation 15 for the extreme anomalous skin effect heating per unit length that is similar to Equation 13 for the ordinary skin effect heating, takes the following form;

$$
\frac{P}{\ell}=\Gamma\left(\frac{5}{6}\right) \frac{3^{1 / 6}}{8 \pi^{2}(2 \pi)^{1 / 3}} \frac{C_{M} c^{2 / 3} \mu^{2 / 3}(\rho \lambda)^{1 / 3}}{R L_{B}^{5 / 3}} \frac{I^{2}}{m},
$$

where $\Gamma(5 / 6)=1.1288$ and everything else is previously defined. 
The constant term in Equations 15 and 16 is 0.0093045. One should note that in Equations 15 and $16, \rho \lambda$ is a constant regardless of the temperature of the beam tube. This suggests that the value of $\rho \lambda$ given by Equation 15 is the lowest value of beam heating per unit length that one can achieve in a cold undulator bore. The $\rho \lambda$ product is only a function of the material that lines the beam tube. Of the materials shown in Table 2, aluminum has the lowest value of $\rho \lambda$. Since the $\rho \lambda$ product taken to the one-third power, the advantage gained from using aluminum is not very great. From Table 2, it is clear that there is no real gain in using silver or gold as the lining material for an undulator beam tube.

Table 3 compares the beam heating per unit length for three types of aluminum and two types of copper at various temperatures for a machine with the following parameters;

$\begin{array}{lc}\text { Machine Circumference } & 300.0 \mathrm{~m} \\ \text { Number of Beam Bunches } & 330 \\ \text { Beam Bunch Length } & 4.0 \mathrm{~mm} \\ \text { Beam Current } & 1.0 \mathrm{~A} \\ \text { Half Gap Radius } & 2.5 \mathrm{~mm} \\ \text { Machine Frequency } \mathrm{f}_{0} & 500 \mathrm{MHz} \\ \mathrm{z}_{\mathrm{o}} & 120 \pi \Omega \mathrm{m}^{2}\end{array}$

Table 3. The Effect of Liner Material and Beam Tube Temperature on Beam Heating per Unit Length

\begin{tabular}{|c|c|c|c|c|c|c|}
\hline Material & $\begin{array}{c}\text { Temperature } \\
\text { (K) }\end{array}$ & $\begin{array}{c}\rho \\
(\Omega \mathrm{m})\end{array}$ & $\alpha$ & $\begin{array}{c}\mathrm{P} / \mathrm{L} \mathrm{Eq.} 14 \mathrm{~b} \\
\left(\mathrm{~W} \mathrm{~m}^{-1}\right)\end{array}$ & $\begin{array}{l}\mathrm{P} / \mathrm{L} \text { Eq. } 15 \\
\left(\mathrm{~W} \mathrm{~m}^{-1}\right)\end{array}$ & $\begin{array}{l}\mathrm{P} / \mathrm{L} \text { est. } \\
\left(\mathrm{W} \mathrm{m}^{-1}\right)\end{array}$ \\
\hline $\mathrm{Cu}, \mathrm{RRR}=300$ & 300 & $1.74 \times 10^{-8}$ & 0,000239 & 80.81 & 15.24 & 80.8 \\
\hline $\mathrm{Cu}, \mathrm{RRR}=300$ & 40 & $2.95 \times 10^{-10}$ & 49.03 & 10.52 & 15.24 & 21.3 \\
\hline $\mathrm{Cu}, \mathrm{RRR}=300$ & 4.2 & $5.53 \times 10^{-11}$ & 7443 & 4.56 & 15.24 & 16.7 \\
\hline $\mathrm{Cu}, \mathrm{RRR}=10$ & 300 & $1.81 \times 10^{-8}$ & 0.000212 & 82.42 & 15.24 & 82.4 \\
\hline $\mathrm{Cu} \mathrm{RRR}=10$ & 40 & $1.88 \times 10^{-9}$ & 0.189 & 26.56 & 15.24 & 26.7 \\
\hline $\mathrm{Cu}$ RRR $=10$ & 4.4 & $1.81 \times 10^{-9}$ & 0.212 & 26.06 & 15.24 & 26.2 \\
\hline $\mathrm{Al}, \mathrm{RRR}=3000$ & 300 & $2.68 \times 10^{-8}$ & 0.000024 & 100.29 & 12.92 & 100.3 \\
\hline $\mathrm{Al}, \mathrm{RRR}=3000$ & 40 & $2.10 \times 10^{-10}$ & 50.39 & 8.88 & 12.92 & 18.0 \\
\hline $\mathrm{Al}, \mathrm{RRR}=3000$ & 4.4 & $\sim 9.0 \times 10^{-12}$ & 640139 & 1.84 & 12.92 & 13.3 \\
\hline $\mathrm{Al}, \mathrm{RRR}=14^{*}$ & 300 & $2.82 \times 10^{-8}$ & 0.000021 & 102.87 & 12.92 & 102.9 \\
\hline $\mathrm{Al}, \mathrm{RRR}=14^{*}$ & 40 & $2.40 \times 10^{-9}$ & 0.0338 & 30.01 & 12.92 & 30.1 \\
\hline $\mathrm{Al}, \mathrm{RRR}=14^{*}$ & 4.4 & $2.01 \times 10^{-9}$ & 0.0575 & 27.47 & 12.92 & 27.6 \\
\hline 6061-T6 Al & 300 & $3.95 \times 10^{-8}$ & 0.000008 & 121.75 & 12.92 & 121.8 \\
\hline 6061-T6 Al & 40 & $1.50 \times 10^{-8}$ & 0.000138 & 75.03 & 12.92 & 75.1 \\
\hline 6061-T6 Al & 4.4 & $1.40 \times 10^{-8}$ & 0.000170 & 72.48 & 12.92 & 72.5 \\
\hline
\end{tabular}


The estimated heat leak per unit length shown in Table 3 for values of $\alpha$ greater than 3 was obtained using the expression given in Equation 10. One can see that when $\alpha$ is about 50, the heating per unit length is about 40 percent higher than the minimum value given by the extreme anomalous heating equation (Equations 15 or 16).

From Table 3, it is clear that bore tubes made from aluminum may be very attractive at low temperature. Aluminum beam tubes must be 20 percent thicker than stainless steel beam tubes (with a copper coating on the inside), but they are quite smooth. The quality of the surface on the inside of the beam tube has a marked effect on the anomalous skin effect heating. A rough surface on the inside of the beam tube can increase the minimum skin effect heating by up to a factor of two.

At intermediate temperatures, (say 30 to $50 \mathrm{~K}$ ) one should strongly consider using a extruded aluminum beam tube made from a material such as 1100-O aluminum that has been annealed. The aluminum beam tube must be welded to a thin wall 5456 aluminum section at the ends in order to minimize the heat flow into the cooled beam tube from the ends. If a 6061 aluminum beam tube can be clad with ultra pure (99.999 percent pure) annealed RRR > 1000 aluminum, one will get minimum beam heating at low temperatures (say less than $30 \mathrm{~K}$ ).

The choice of beam tube material is clearly a compromise between beam heating and the bore tube thickness (thus the $\mathrm{y}$ dimension) that can be achieved. At temperatures from $300 \mathrm{~K}$, to $30 \mathrm{~K}$ an aluminum bore tube is attractive when the beam heating is high. When the bore tube temperature is below $30 \mathrm{~K}$, one should go to a bore that is clad with pure copper or pure aluminum. The only argument in favor of using gold or silver as a cladding material is that these materials may improve the quality of the bore tube surface.

\section{Heat Flow into the Cryostat by Conduction and Thermal Radiation}

The physical size of the insertion device coils is directly related to the physical cold magnet gap $G_{M}$ defined by equation 3. The cold mass for the magnet is proportional to the length of the magnet $\mathrm{L}_{\mathrm{M}}$ and $\mathrm{G}_{\mathrm{M}}$ squared. For a superconducting coil system similar to that shown in Figure 6, the cold mass for the insertion magnet may be estimated using the following general expression;

$$
M_{C}=\rho_{F e} \frac{\pi}{8}\left(100 G_{M}^{2}\right) L_{M}+50
$$

where $M_{C}$ is the magnet cold mass; $\rho_{\mathrm{Fe}}$ is the density of iron $\left(\rho_{\mathrm{Fe}}=7900 \mathrm{~kg} \mathrm{~m}^{-3}\right) ; \mathrm{G}_{\mathrm{M}}$ is the magnet gap defined by Equation 3; and $\mathrm{L}_{\mathrm{M}}$ is the length of the magnet. Figure 6 shows holes in the iron filled with liquid helium. These holes may be larger than shown. The cold mass calculated from Equation 17 includes the cryostat vessel, any necks, and the cold mass support connectors.

Once one has estimated the cold mass for the magnet, one can estimate the conduction heat leak into the magnet and the cold mass support intercepts. In general, the heat leak is proportional to the cold mass, thermal conductivity integral for the cold mass support strut material, the area of the cold mass support struts. The conduction heat leak is inversely proportional to the length of the support struts. A simple form of the heat leak equation based on measurements of Superbend at LBNL [1] for heat leak from $40 \mathrm{~K}$ to $4.2 \mathrm{~K} \mathrm{Q}_{\mathrm{C} 4.2}$ is as follows;

$$
Q_{C 4.2}=0.00015 M_{C}
$$


The conduction heat leak from $300 \mathrm{~K}$ to about $40 \mathrm{~K} \mathrm{Q}_{\mathrm{C} 40}$ takes the following form;

$$
Q_{C 40}=0.0035 M_{C}
$$

The conduction heat loads given in Equations 19 and 20 include heat leaks down the support bands, cryogenic necks and the wires used for instrumentation of the magnet and its cryogenic system. The values given by Equations 18 and 19 are approximate. The largest portion of the values given above represent the heat leak down cryogenic necks. The thinner the neck the lower its heat leak. Cryogenic necks must be fabricated from a low heat leak material such as 304 stainless steel or titanium. In most cases gas cooling of the cryostat cryogenic neck is not an option.

The surface area $\mathrm{A}_{\mathrm{S}}$ for the insertion-device magnet cold mass can be estimated using the following expression;

$$
A_{S}=\frac{\pi}{4}\left(100 G_{M}^{2}\right)+60 G_{M} L_{M}
$$

The design radiation and conduction heat load through the multi-layer insulation (MLI) from 40 or $50 \mathrm{~K}$ to $4.2 \mathrm{~K} \mathrm{Q}_{\mathrm{R} 4.2}$ can be calculated using the following approximate expression;

$$
Q_{R 4.2}=0.05 A_{s}
$$

The design radiation and conduction heat load through the MLI from $300 \mathrm{~K}$ to $40 \mathrm{~K} \mathrm{Q}_{\mathrm{R} 40}$ can be calculated using the following approximate expression;

$$
Q_{R 40}=2.0 A_{S}
$$

The radiation heat loads given by equations 21 and 22 are approximate. With care one can improve on these values by a factor of 1.5 to 2 . If liquid helium or liquid nitrogen tanks are included with the cold mass, the area of the tanks should be included with the surface area of the magnet. One must take great care to see that there is multi-layer insulation between room temperature and all $4 \mathrm{~K}$ surfaces.

\section{Heat Transfer into the Cryostat due to the Magnet Leads}

The electrical leads for the magnet can be the largest heat load into a $4.4 \mathrm{~K}$ cryostat. Since the heat lead from the magnet leads can be high, magnets that are cooled using small two stage cryo-coolers will always have high temperature superconductor (HTS) leads between the magnet (at $4.4 \mathrm{~K}$ ) and the first stage of the cooler, which is usually at 40 to $70 \mathrm{~K}$. Magnets that are cooled are cooled with a larger refrigerator, that has a J-T that produces two-phase helium, will usually have leads that are cooled using helium that is boiled in the cryostat. This gas is returned to the refrigerator compressor intake at room temperature.

One can estimate the direct heat leak into the magnet (at $4.4 \mathrm{~K}$ ) if one knows the lead current I and the cryo-cooler first stage temperature $\mathrm{T}_{\mathrm{cl}}$. When HTS leads are used between $\mathrm{T}_{\mathrm{cl}}$ and $4 \mathrm{~K}$, the following approximate equation can be used to calculate the heat load; 


$$
Q_{T_{c 1}-4}=0.3\left(\frac{T_{c 1}}{60}\right)^{2}\left(\frac{I}{300}\right)
$$

The lead heat leak calculated using Equation 23 applies when the leads carry current and when the current is shut off.

The heat leak down the leads from $300 \mathrm{~K}$ to the first stage of the cooler when the leads carry their design current I can be estimated using the following expression;

$$
Q_{300-T_{c 1}}=0.081 I \quad-24 \mathrm{a}-
$$

The heat leak down the leads from $300 \mathrm{~K}$ to the first stage of the cooler when the leads do not carry current can be estimated using the following expression;

$$
Q_{300-T_{c 1}}=0.027 I
$$

$-24 b-$

When the leads carry a current below their design current I, the heat load to the first stage of the cooler is somewhere between the extremes shown in Equations 24a and 24b. When the magnet design current is large, there is an incentive to operate the magnet in persistent mode, in order to reduce the heat leak into the first stage of the cooler. Retractable copper leads also make a great deal of sense because the heat flow into the first stage is reduced and along with the reduction of the first stage heat load there is a reduction in the cooler first stage temperature.

When a wiggler or undulator is cooled using a standard helium refrigerator with a J-T circuit to make liquid helium, the leads that connect the superconducting magnet with the outside world are often gas-cooled, using helium from either a two-phase helium stream or boil off gas from the magnet cryostat. If the gas-cooled leads are properly designed, the net heat flow into the $4 \mathrm{~K}$ region can be reduced to almost zero. The refrigeration needed to cool the leads comes from the fact that the gas used to cool the electrical leads does not return to the refrigerator compressor through the refrigerator heat exchanger. This gas represents a virtual liquefaction (when helium is liquefied it does not return to the compressor through the heat exchanger).

The design gas flow up the leads $\mathrm{m}_{\mathrm{g}}$ needed to intercept virtually all of the heat coming down a pair of gas cooled leads can be estimated using the following expression, when the leads carry their design current [14];

$$
m_{g}=0.9 \times 10^{-7} I \rightarrow 1.1 \times 10^{-7} I
$$

where the gas mass flow per lead pair $\mathrm{m}_{\mathrm{g}}$ is given in $\mathrm{kg} \mathrm{s}^{-1}$ and the current $\mathrm{I}$ is given in $\mathrm{A}$. The gas flow through the lead goes down when the lead is operated below its design current and the gas flow goes up when the lead is operated above its design current. In general, the lead mass flow can be controlled by measuring the voltage drop across the lead. Once one knows the lead gas mass flow one can estimate the amount of the helium refrigeration needed to cool a pair of leads. The following expression can be used to estimate the amount of $4.2 \mathrm{~K}$ refrigeration $\mathrm{Q}_{\mathrm{L}}$ needed to cool the gas cooled leads that are cooled using a large refrigerator with a J-T circuit; 


$$
Q_{L}=\Xi_{R L} m_{g}
$$

where $\Xi_{R L}$ is the refrigeration to liquefaction coefficient for the helium refrigerator that is being used to cool the superconducting magnet. Most helium refrigerators can be used as either refrigerators or as helium liquefiers. Each of these machines has a characteristic value for the refrigeration to liquefaction coefficient $\Xi_{\mathrm{RL}}$. Typical machines have values of $\Xi_{\mathrm{RL}}$ that range from 85 to $125 \mathrm{~kJ} \mathrm{~kg}^{-1}$. (If one is to use a single value, $\Xi_{\mathrm{RL}}=100 \mathrm{~kJ} \mathrm{~kg}^{-1}$.) In round number terms about $10 \mathrm{~W}$ of refrigeration is needed to cool a pair of leads for each $\mathrm{kA}$ of lead design current. When there is no current in the leads, the refrigeration needed to cool the leads may be reduced by as much as 40 percent.

\section{CRYOGENIC COOLING OPTIONS}

\section{To use a Cryo-cooler or not to use a Cryo-cooler, that is the Question}

The use of a cryo-cooler is dictated by the heat loads at $4 \mathrm{~K}$ second stage and at the first stage temperature of the machine (heat loads on the shields and intercepts plus the heat load on an intermediate temperature bore tube plus the conduction heating from leads). Experience at LBNL has shown that multiple coolers can be used to cool a device provided one connects the cooler (coolers) to the load in a way that ensures a low temperature drop between the magnet coils and the cooler second stage cold head. Methods for connecting the cooler to the load will be discussed later in this report.

The upper practical limit for the heat load at 4.2 to $4.4 \mathrm{~K}$ when one uses cryo-coolers to cool the magnet is about 5 watts. This means that three Sumitomo Gifford McMahon (GM) $1.5 \mathrm{~W}$ (at $4.2 \mathrm{~K}$ ) coolers can be used, or one can up to five Cryomech $0.9 \mathrm{~W}$ (at $4.2 \mathrm{~K}$ ) pulse-tube coolers to cool a single Insertion magnet (see Figure 7). The maximum number of coolers that one would use to cool a device is arbitrary, so the numbers given here should be regarded as preliminary. In general, the physical size of the device or its mass is not a dominant factor in the selection of the cooler. The Superbend magnets, which have a cold mass of $1700 \mathrm{~kg}$ and a surface area of nearly $10 \mathrm{~m}^{2}$ are cooled using a single $1.5 \mathrm{~W}$ cooler [15],[16]. A number of factors come into play when one is selecting the number of coolers that might be used to cool a single device. These factors include the cost of each cooler and the proximity of the device to another device that is cooled to liquid helium temperature.

The amount of cooling required at 4.2 to $4.5 \mathrm{~K}$ is not the only factor that determines whether the device can be cooled using one or more coolers. The heat load at say $50 \mathrm{~K}$ is also a very important factor. The upper limit for the heat load into the device at $50 \mathrm{~K}$ is about $150 \mathrm{~W}$. The heat load into an

insertion device magnet cooled with one or more cryo-coolers is dominated by the upper leads. Even if the heat load at 4.2 to $4.5 \mathrm{~K}$ is below $5 \mathrm{~W}$, one can not effectively cool a device that operates continuously at a total current of over $1700 \mathrm{~A}$ (maybe $3500 \mathrm{~A}$ if there is a liquid nitrogen cooled point on the upper leads that are cooled by conduction). If one can operate the insertion device in persistent mode, the upper limit for the total current into the device may approach 6 to $8 \mathrm{kA}$. 


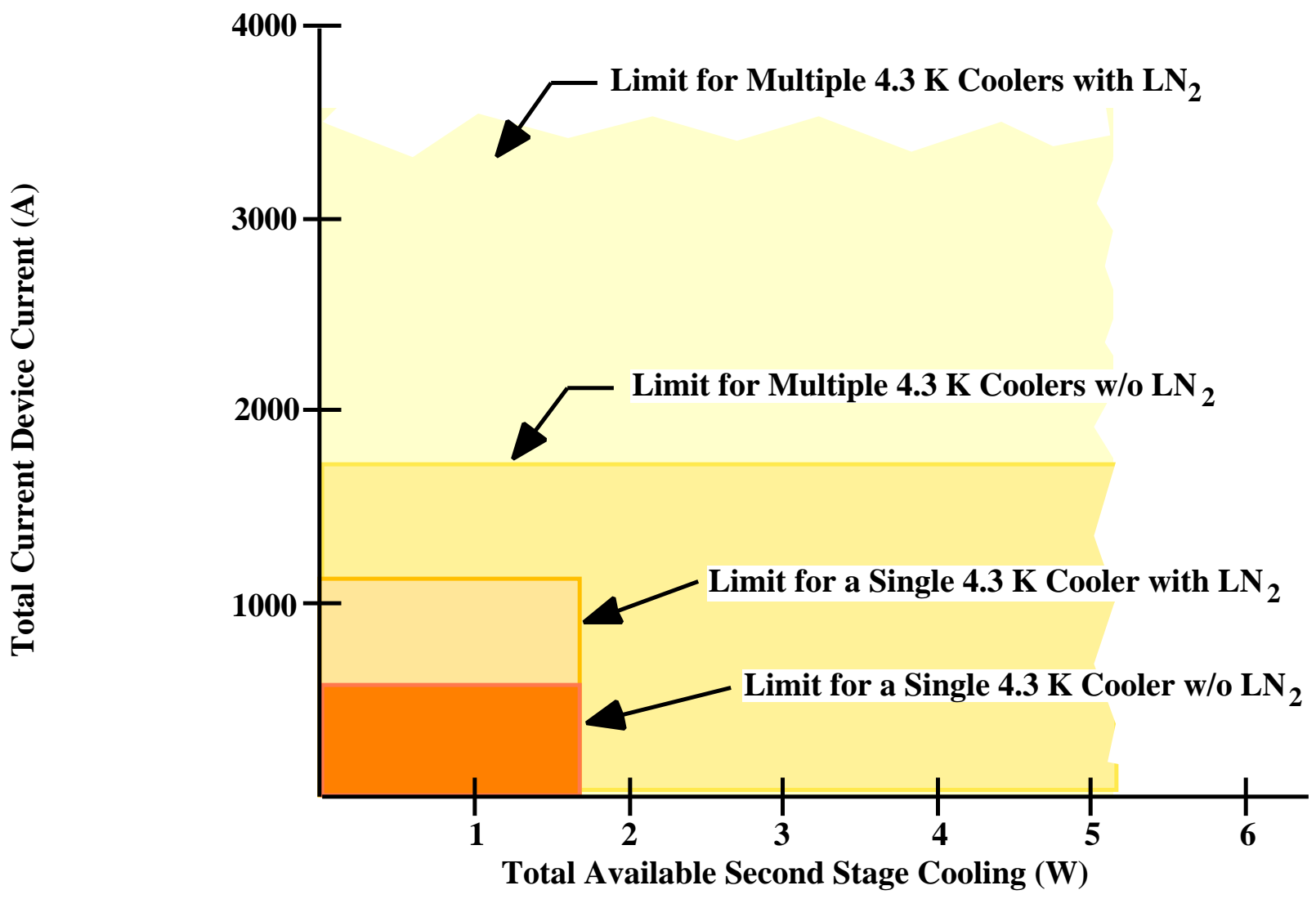

Figure 7. Practical Heat Load and Current Limits for cooling Insertion Devices with One or More Small Coolers The orange regions represent the limit for a single 1.5 W GM cooler; the yellow regions represents a practical limit for cooling an insertion device with multiple coolers. Liquid nitrogen can be used to intercept heat from $300 \mathrm{~K}$, thus extending the total current applicability into the device when it is cooled using small coolers. In all cases, it is assumed that beam heating on a bore tube (at $\mathrm{T}>35 \mathrm{~K}$ ) can be removed by one or two single stage pulse tube coolers.

The mass and surface area of an insertion device are not a primary factors that affect the heat load into the first stage of the cooler. Lead cooling and intermediate temperature bore tube cooling are more important in determining the amount of cooling that must be applied by the first stage of a $4.2 \mathrm{~K}$ cooler. If one is willing to use liquid nitrogen to cool the upper leads, the region of applicability for small coolers can be extended toward higher total currents powering the device.

Cooling at the ends of a intermediate temperature insertion device bore can be supplied by a pair of separate low-vibration pulse-tube coolers provided the amount of cooling needed by the cold beam vacuum tube does not exceed about $80 \mathrm{~W}$. An illustration of the limits over which one may use one or more cryo-coolers to cool an insertion device is illustrated in Figure 7. Outside of the yellow region shown in Figure 7, one must use a larger refrigerator (>15 W at $4.4 \mathrm{~K}$ ) with a final stage of J-T expansion, so that leads and shields can be gas-cooled. Cooling of insertion magnets using stored liquid cryogens is always an option, but in the long run it is not an option that makes economic sense. If the heat load (at any temperature) and current in the insertion device are too high to use one or more small coolers, a large helium refrigerator with a final J-T expansion should be used to cool the device. 


\section{Candidate Cryogenic Coolers for cooling Insertion Devices}

At the time this report was completed (August 2003), there were only two cryogenic coolers that one would consider for cooling superconducting insertion devices that operate at 4.2 to $4.4 \mathrm{~K}$ with either niobium titanium or niobium tin as the conductor used in the magnet coils. The first cooler is the two stage Gifford McMahon (GM) cycle cooler made by Sumitomo (the RDK-415D cooler) that develops $1.5 \mathrm{~W}$ of cooling at $4.2 \mathrm{~K}$ while delivering $63 \mathrm{~W}$ of cooling at $60 \mathrm{~K}$. Sumitomo also makes a $0.1 \mathrm{~W}$ cooler, a $0.5 \mathrm{~W}$ cooler, and $1.0 \mathrm{~W}$ cooler that operate using the same refrigeration cycle. The smaller coolers deliver proportional less cooling at $60 \mathrm{~K}$. The second suggested cooler is the two stage pulse tube (PT) cooler made by Cryomech (the PT407 cooler) that develops $0.7 \mathrm{~W}$ of cooling at $4.2 \mathrm{~K}$ while delivering $38 \mathrm{~W}$ of cooling at $60 \mathrm{~K}$. Cryomech also makes a $0.5 \mathrm{~W}$ pulse tube cooler. A version of their PT407 cooler that develops $0.9 \mathrm{~W}$ at $4.2 \mathrm{~K}$ will soon become available. The refrigeration at $60 \mathrm{~K}$ is proportional to the refrigeration produced at $4.2 \mathrm{~K}$. The Sumitomo cooler is recommended because it is the largest cooler available for use on a magnet. The Cryomech cooler is recommended because of its low vibration and high reliability. (There are no moving parts in the cold head.)

Many single stage GM and pulse tube coolers are available that can be used in the temperatures range between 40 and $70 \mathrm{~K}$. The cooler that the author recommends (in August 2003) is the Cryomech PT60 cooler. This cooler develops $18 \mathrm{~W}$ at $40 \mathrm{~K}, 33 \mathrm{~W}$ at $50 \mathrm{~K}$, and $60 \mathrm{~W}$ at $80 \mathrm{~K}$. This cooler is recommended because of its low vibration and high reliability. (There are no moving parts in the cold head.) Table 4 compares various parameters for the recommended cryogenic coolers.

Table 4. A comparison of the Recommended $4 \mathrm{~K}$ and $50 \mathrm{~K}$ Cryogenic Coolers for Insertion Devices

Parameter

Type of Refrigeration Cycle

Number of Cooling Stages

Lowest Temperature for the $1^{\text {st }}$ Stage (K)

Lowest Temperature for the $2^{\text {nd }}$ Stage $(\mathrm{K})$

Available Cooling at $60 \mathrm{~K}^{*}(\mathrm{~W})$

Available Cooling at $4.2 \mathrm{~K}(\mathrm{~W})$

Input Power to the Compressor $(\mathrm{kW})$

Cooling Water Required (liters/min)

Time to Cool-down the Cooler Alone (min)

Cold Head Mass (kg)

Compressor Mass (kg)

Cold Head Vibration Acceleration $\left(\mathrm{m} \mathrm{s}^{-2}\right)$

Vibration Force Amplitude at Cold Head (N)

Primary Vibration Frequency $(\mathrm{Hz})$

Maintenance Interval for the Cold Head (hr)

$\begin{array}{ccc}\begin{array}{c}\text { Sumitomo } \\ \text { RDK-415D }\end{array} & \begin{array}{c}\text { Cryomech } \\ \text { PT407 }\end{array} & \begin{array}{c}\text { Cryomech } \\ \text { PT60 }\end{array} \\ \text { GM } & \text { PT } & \text { PT } \\ 2 & 2 & 1 \\ \sim 23 & \sim 26 & \sim 28 \\ \sim 2.8 & \sim 2.2 & -\mathrm{NA}- \\ 63 & 38 & 45 \\ 1.5 & 0.7 & -\mathrm{NA}- \\ 7.5 & 7.0 & 3.0 \\ 7 & 7 & 3 \\ <100 & <60 & <30 \\ 7.2 & 14.0 & 6.8 \\ 115 & 119 & 52.2 \\ <0.9 & <0.01 & <0.01 \\ \sim 6.4 & \sim 0.13 & \sim 0.07 \\ \sim 20 & \sim 3 & \sim 5 \\ 10000 & >50000 & >50000 \\ \text { a } 50 \mathrm{~Hz} \text { motor, the refrigeration developed at } 60 \mathrm{~K} \text { is reduced. }\end{array}$


Figure 8 shows the standard operating diagram for the Sumitomo RDK-415D cryo-cooler [17]. This machine uses a high heat capacity regenerator in the second stage cold head in order to achieve operating temperatures as low as $2.8 \mathrm{~K}$. Figure 9 and 10 show measured cooling data for one of the four RDK-415D machines used to cool one of the Superbend dipoles at the ALS [18]. The data in Figure 10 does not show the lower operating temperature on the second stage when the first stage is fully loaded. The reason for this is that the LBNL test also tested the HTS lead as well as the cooler. The heat flow down the HTS leads increases as the first stage temperature goes up. The improvement of machine performance is counteracted by the additional heat leak into the second stage through the HTS leads. The experimental data shown in Figures 9 and 10 may be more indicative of the performance of the cooler on a magnet. The measured temperature scatter shown in Figure 10 may be do to the temperature oscillation in the second stage cold head. This oscillation peak to peak amplitude can be as large $0.3 \mathrm{~K}$

Both the first and seconds stages of the RDK-415D GM cooler have moving pistons within the cold heads. The moving pistons in the cold heads are a cause of the larger vibration accelerations seen on the cold head surfaces of this machine. The recommended maintenance interval on the RDK-415D cold head is 10000 hours. This interval must be honored if one wants the machine to operate reliably over a period of years. Having a spare machine on site is recommended if one needs to operate the machine on an insertion device 24 hours a day for many years. The compressors for this machine are quite reliable, bur regular maintenance of the compressors is also required for long term operation.

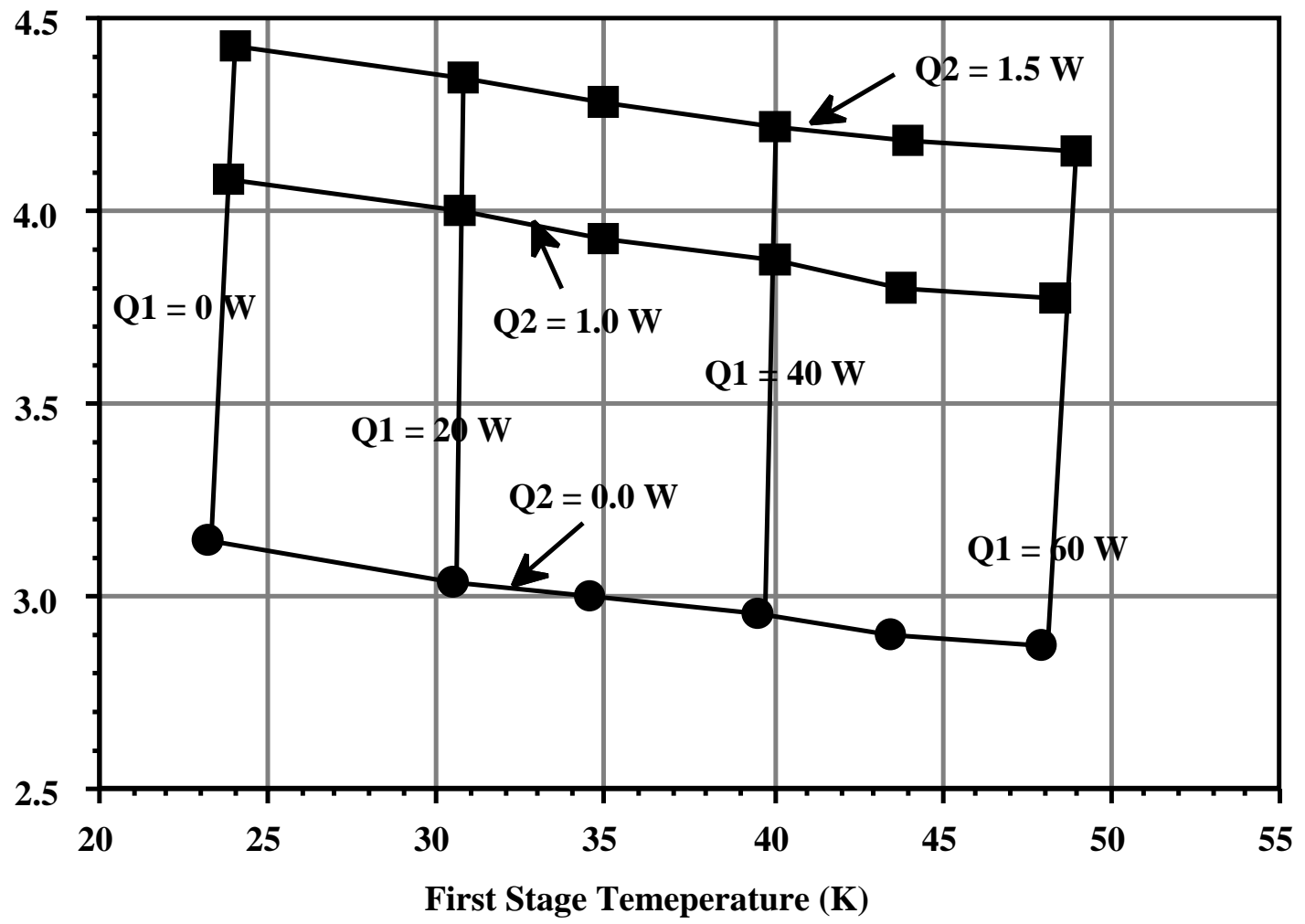

Figure 8. The Standard Operating Performance Diagram for the Sumitomo RDK-415D Gifford McMahon Cooler Note: at $5 \mathrm{~K}$ this cooler will remove about $2.4 \mathrm{~W}$ of heat; at $7 \mathrm{~K}$ this cooler can remove about $5 \mathrm{~W}$ of heat. 


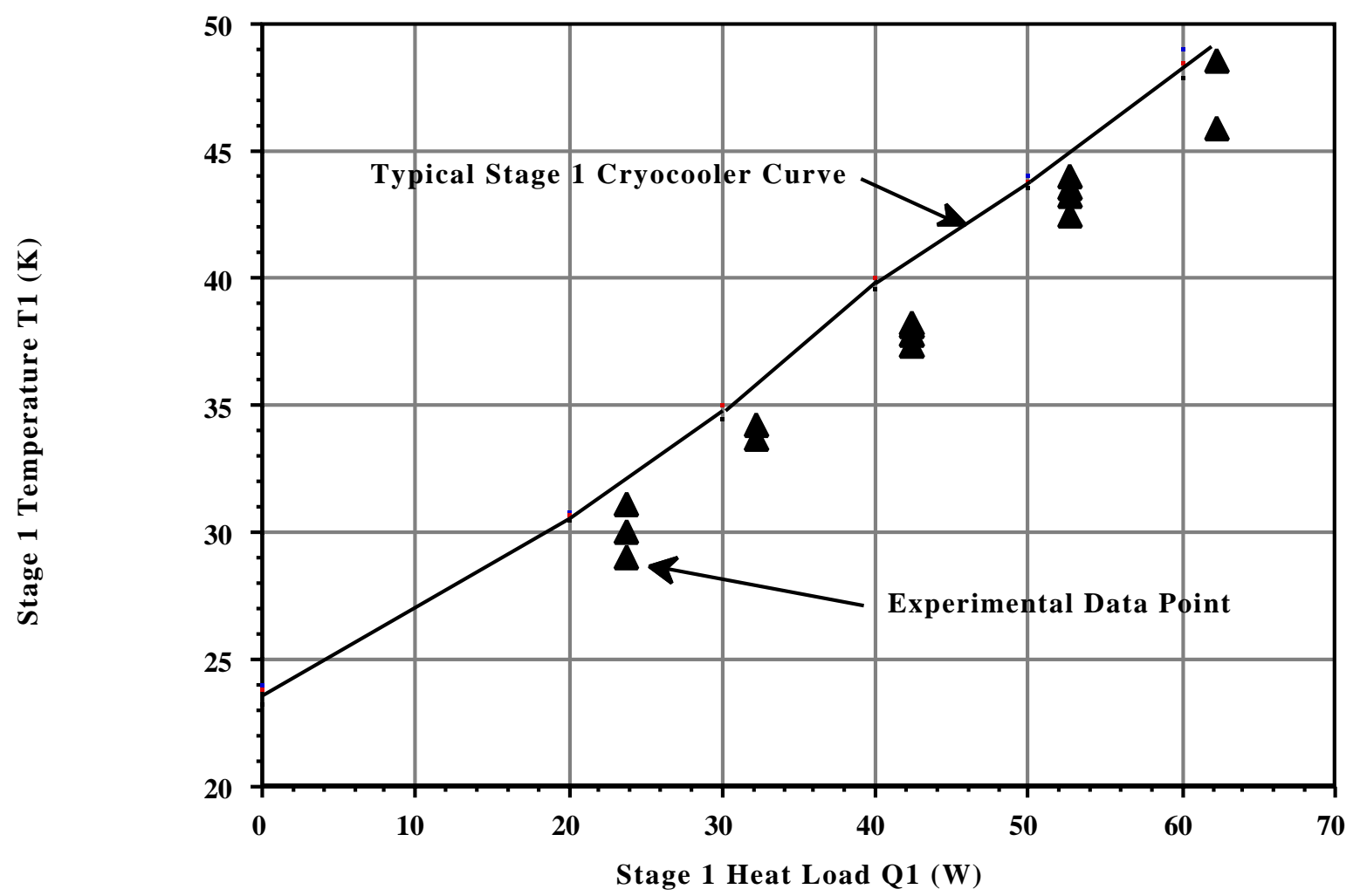

Figure 9. Measured First Stage Temperature T1 as a Function of First Stage Heat Load Q1 for the RDK-415D Cooler

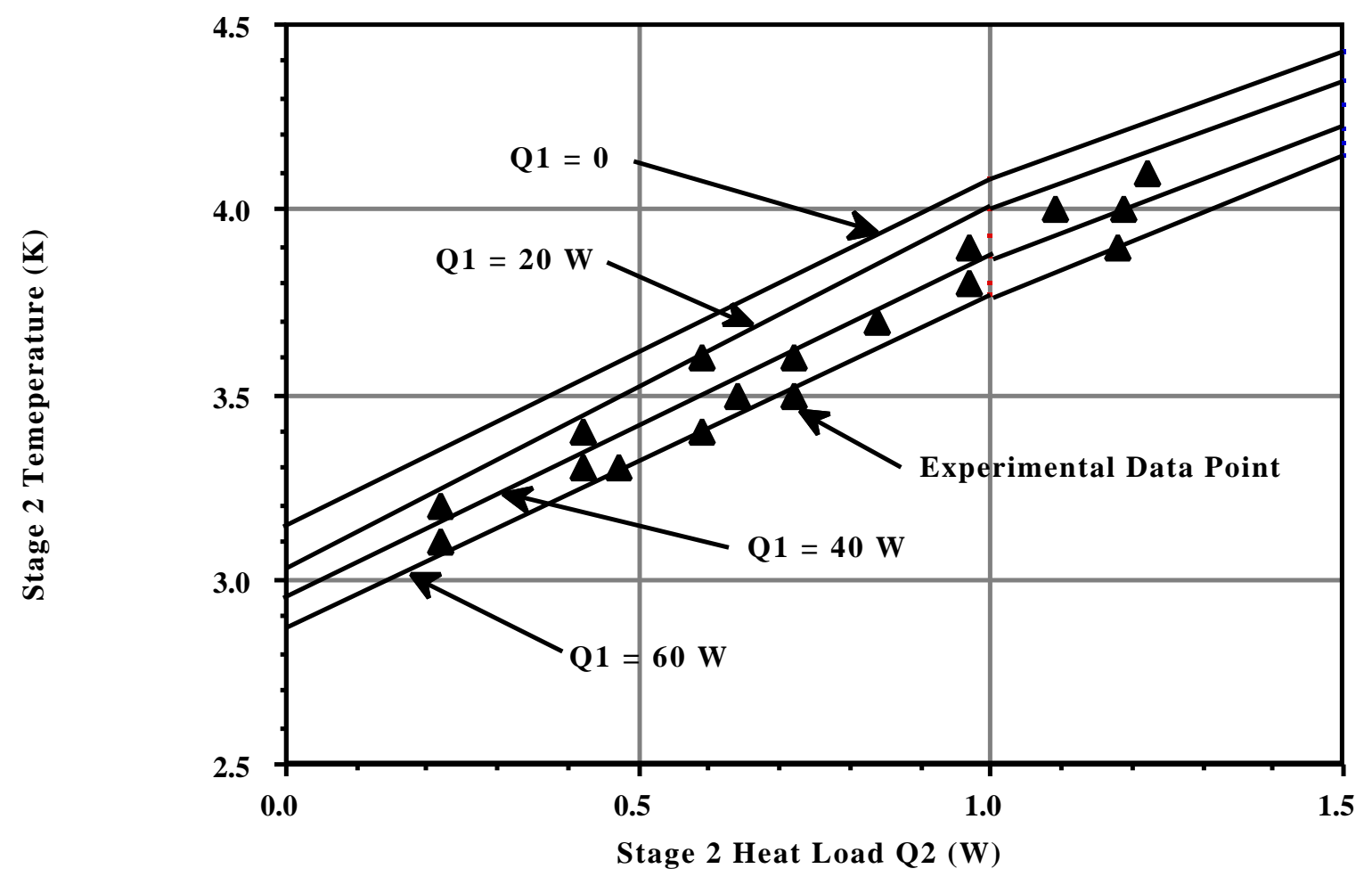

Figure 10. Measured RDK-415D Cooler Second Stage Temperature as a Function of Second Stage Heat Load Q2 and Q1 
The performance data for the Cryomech PT407 pulse tube cooler is shown in Figure 11 [19]. At the higher second-stage heat loads, this cooler has a performance curve that is similar to the performance curve for the Gifford McMahon $4 \mathrm{~K}$ cooler that is shown in Figure 8. The temperature of the second stage at high heat load goes down with heat input into the cooler first stage. Unlike the Gifford McMahon cooler, the $0 \mathrm{~W}$ heat load second-stage temperature goes up as heat is put into the first stage of the cooler. As with the GM cooler, the reduced temperature that is observed as the first-stage heat load goes up may be of little value, because the heat load into the second-stage will go up as the temperature of the first stage goes up. Like the GM cooler pulse tube coolers use a high heat capacity regenerator in the second stage cold head. Like the GM machines, there is an oscillation of the second stage temperature. The peak to peak amplitude of the oscillation is similar to the GM cooler.

An important feature of the PT407 pulse tube cooler, is the fact that there are no moving parts in the cooler cold head for either stage. The only moving part is the gas. As a result, vibration accelerations on the cold head are greatly reduced [20]. Since there are no moving parts in the pulse tube cold head, the maintenance interval for the machine is far longer than for a GM machine. It is reasonable to predict that the pulse tube machine will have a much lower cold-head failure rate than a GM machine. Coldhead orientation is more important for pulse tube coolers than with the GM coolers. Care should be taken to see that the pulse tube cooler cold-head is properly oriented.

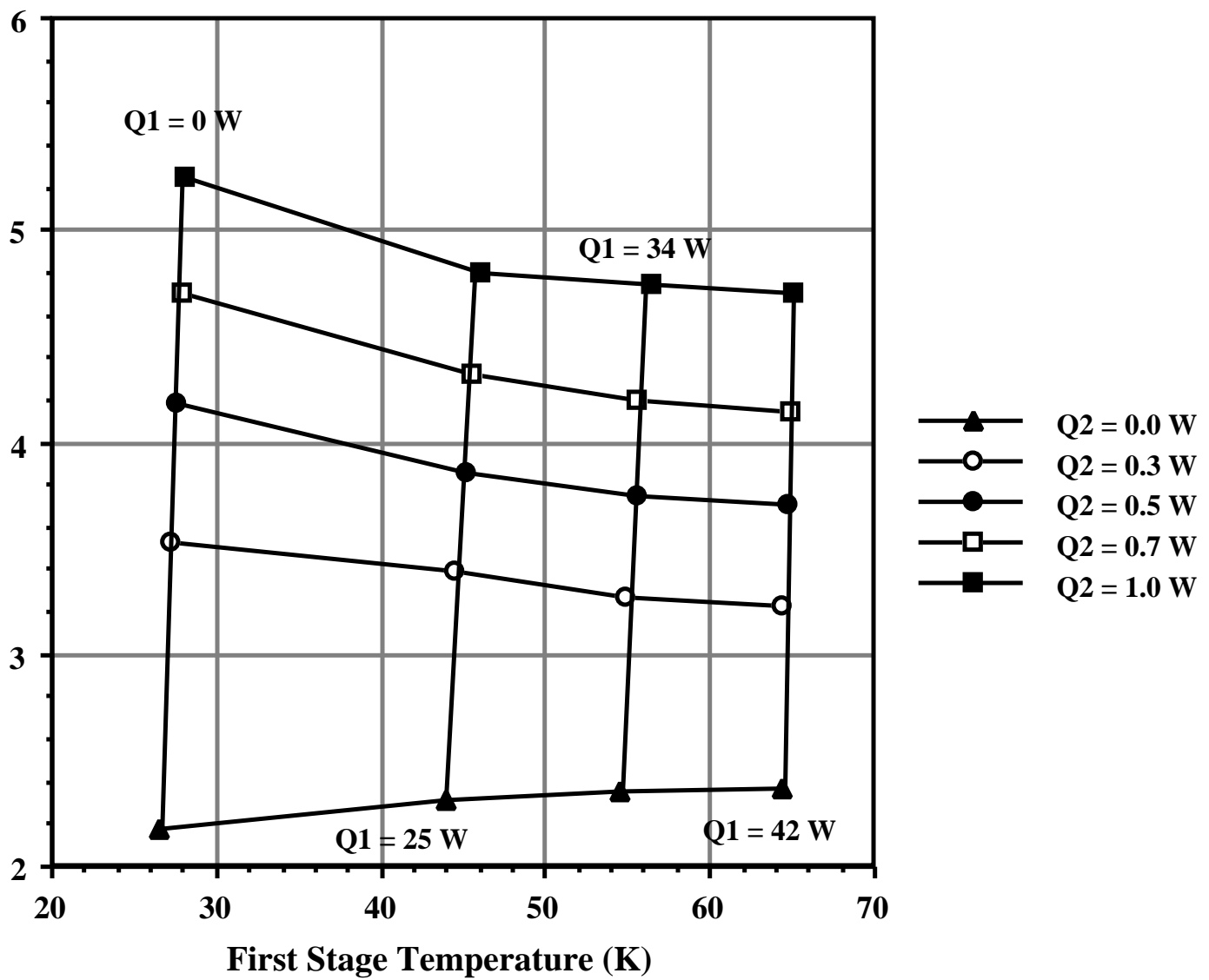

Figure 11. The Standard Operating Performance Diagram for the Cryomech PT-407 Pulse Tube Cooler Note: at $6.3 \mathrm{~K}$ this cooler will remove about $2.0 \mathrm{~W}$ of heat. 
A performance curve for the Cryomech PT-60 cooler is shown in Figure 12 [19]. The performance curve for this cooler is similar in shape to the performance curve for the first-stage of the PT-407 cooler (see Figure 11). In fact the performance curve is too so different than the first-stage performance for the GM cooler (see Figures 8 and 9). The compressor size for this machine is half the size of the PT-407 cooler compressor or the RDK-415D GM cooler compressor. Like the first stage of the two $4 \mathrm{~K}$ machines, the PT-60 cooler develops no cooling below $25 \mathrm{~K}$. Roughly half of the compressor capacity in the two $4 \mathrm{~K}$ coolers is used to produce the $4 \mathrm{~K}$ cooling. The rest of the compressor capacity is used to produce cooling in the temperature range from 30 to $80 \mathrm{~K}$.

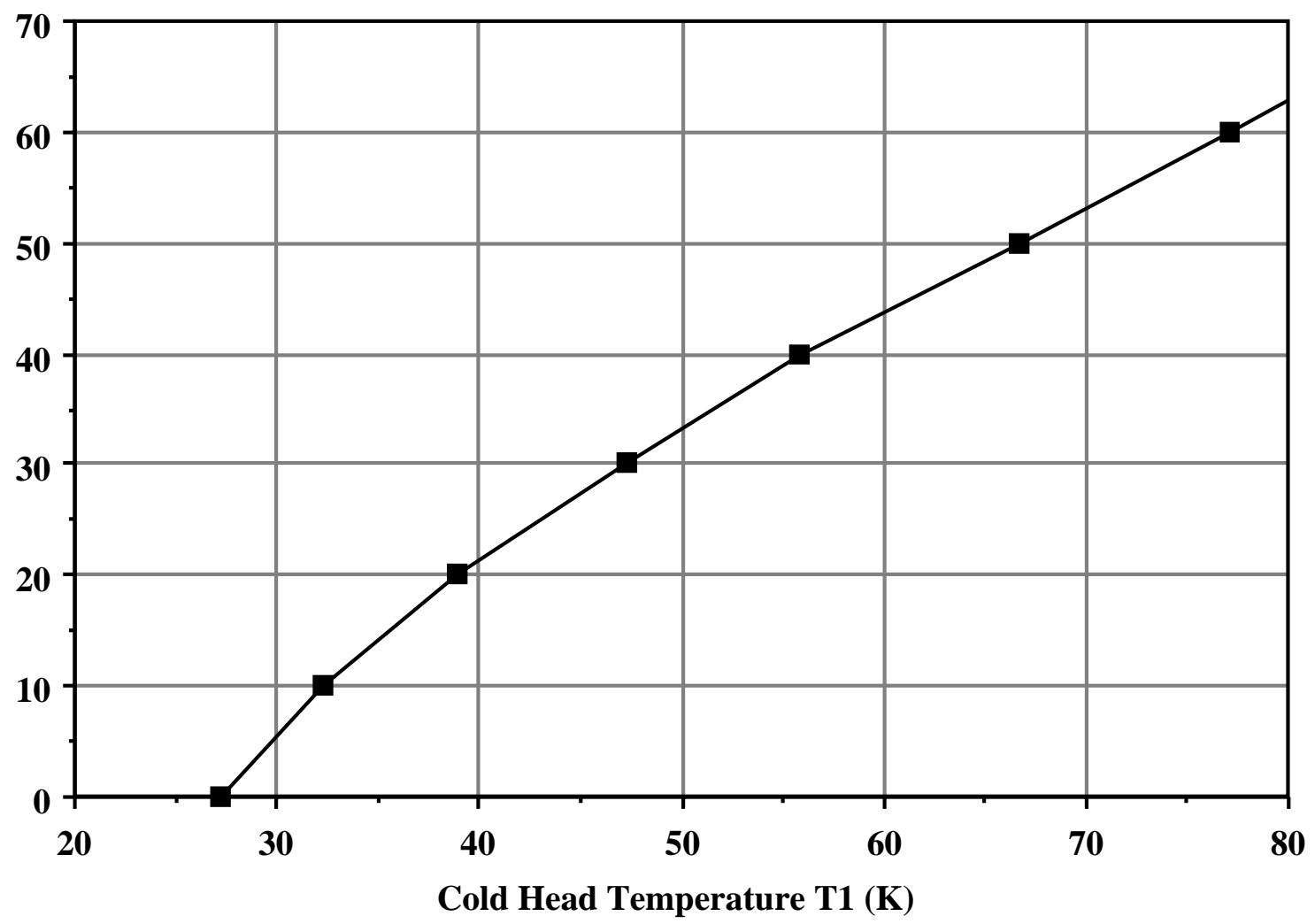

Figure 12. The standard Performance Curve for the Cryomech PT-60 Single Stage Pulse Tube Cooler

PT-60 coolers can be used to produce additional cooling for solid copper leads above $60 \mathrm{~K}$ that is above and beyond the cooling produced by the first stage of the $4 \mathrm{~K}$ cooler. This might allow one to extend insertion device current range shown in Figure 7 while using one or more small cryo-coolers to cool the device.

One or two PT-60 coolers can also be used to cool the cold bore tube of an insertion device as long as that bore temperature is above say $35 \mathrm{~K}$. The PT-60 cooler is an excellent cooler for providing the extra cooling that may be needed for magnet leads or the cold bore of an insertion device. Strong arguments for the use of the PT-60 cooler include; low vibration acceleration $(<0.003 \mathrm{~g})$, no moving parts in the cold head, and long operating time between required maintenance $(>50000 \mathrm{hr})$. The PT-60 and the PT-407 coolers are good machines to use to cool insertion device magnets. 


\section{Insertion Device Cooling using a Conventional Refrigerator}

Insertion devices with parameters that lie out side the range of the colored region shown in Figure 7 must be cooled using a conventional liquid helium refrigerator with a $\mathrm{J}$ - $\mathrm{T}$ valve to provide the liquid helium at $4.4 \mathrm{~K}$. An insertion device with a $4.4 \mathrm{~K}$ bore must always be cooled using a conventional J-T refrigerator. (Small cryo-coolers can not be used to cool an insertion magnet with a $4 \mathrm{~K}$ bore.) From an economic standpoint, cooling with liquid helium does not make sense unless a helium refrigerator is used as a helium liquefier to provide liquid helium to the magnet cryostat. Figure 13 shows a typical bath cooled magnet cryostat that is cooled with a conventional refrigerator. The shields and gas-cooled leads are cooled by cold gas from the magnet cryostat. As an alternative, the shields and cold mass support intercept can be cooled with liquid nitrogen. Cooling for the gas-cooled leads must come from gas in the liquid helium tank.

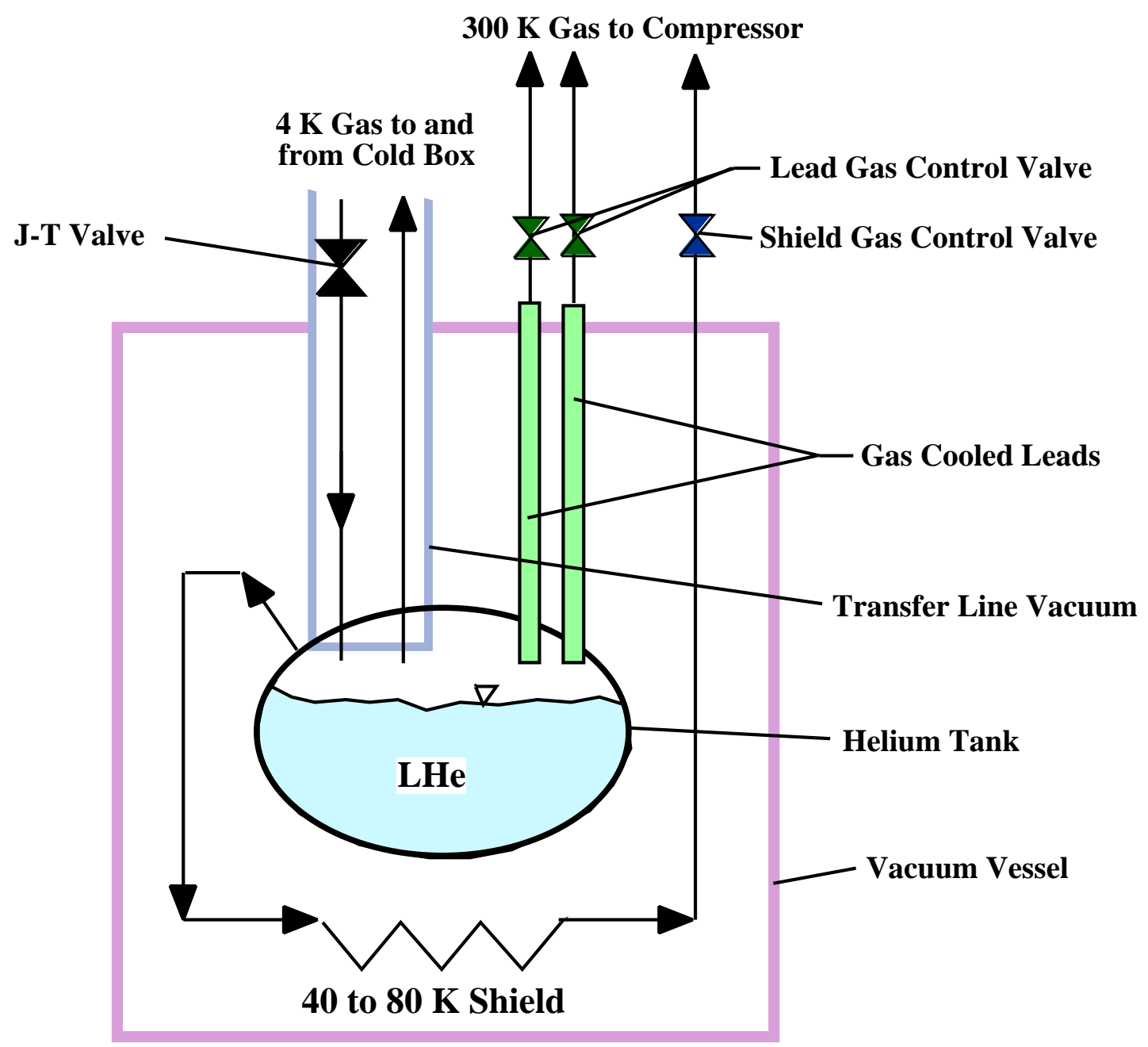

Figure 13. A Conventional Helium Refrigeration System that uses 4 K Helium Gas to Cool the Leads and Shields Note: Equation 25 can be used to estimate the helium mass flow for the leads. The helium mass flow needed to cool the shields can be estimated using the following expression; $\mathrm{m}_{\mathrm{S}}=4 \times 10^{-6} \mathrm{Q}_{\mathrm{S}}$ when the gas leaves the shield and intercepts at $60 \mathrm{~K}$. The equivalent $4.4 \mathrm{~K}$ refrigeration needed to cool the leads and shields can be estimate using Equation 26 provided the value the lead gas mass flow $m_{g}$ is replaced by the total lead and shield mass flow $m_{g}+m_{s}$. 


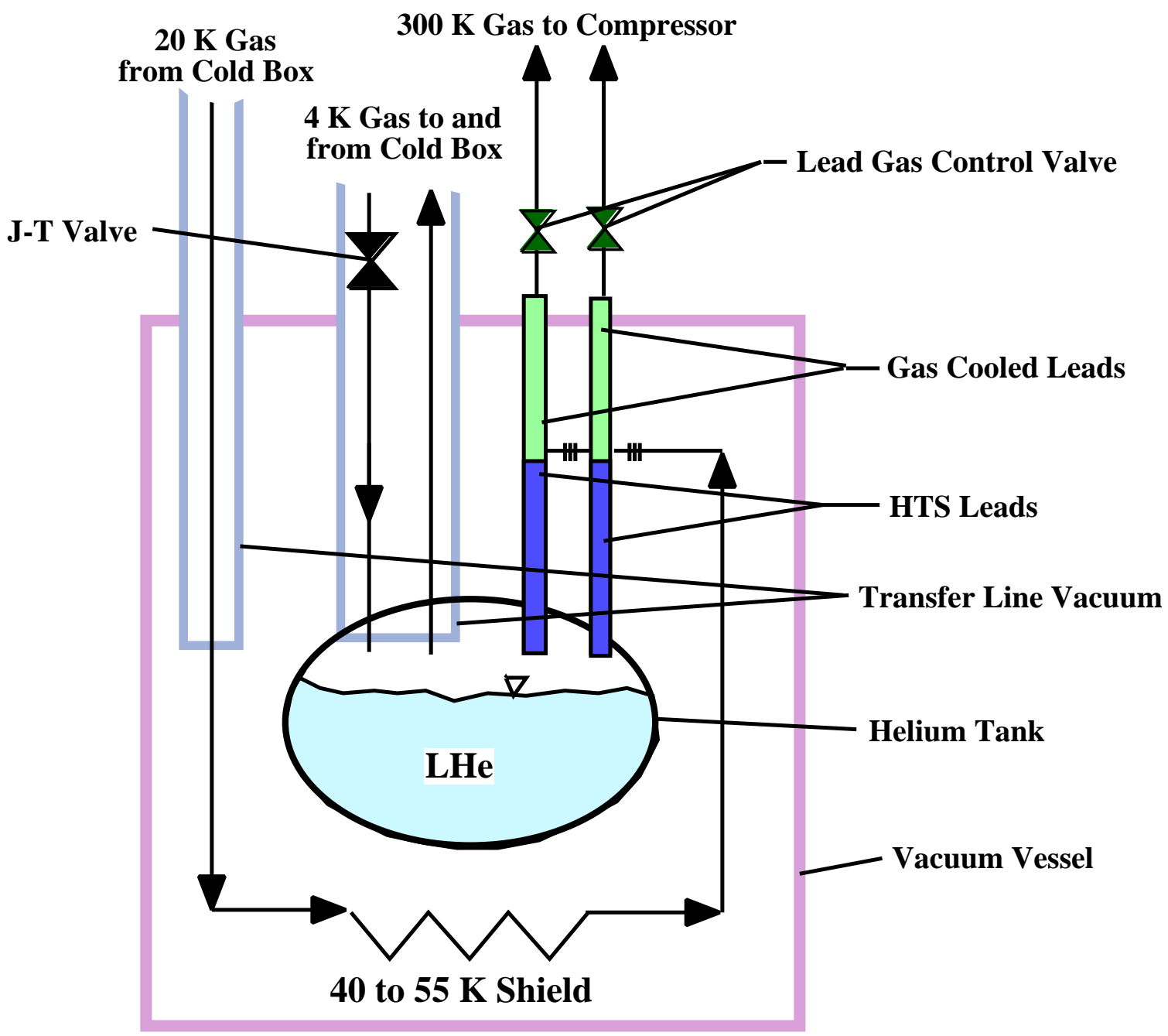

Figure 14. A Conventional Refrigerator System that uses a 20 K Gas Stream to cool Shields and Gas-cooled Leads Note: High temperature superconductor (HTS) leads are shown so that the equivalent $4.4 \mathrm{~K}$ refrigeration can be reduced by a factor of four or more. There are many variations of the flow circuit shown above.

Another alternative is to use a helium refrigerator that delivers two-phase helium at $4.4 \mathrm{~K}$ while delivering helium gas at 20 to $40 \mathrm{~K}$. The gas the 20 to $40 \mathrm{~K}$ stream can be used to cool the magnet shields, cold mass support intercepts, a bore tube at 25 to $40 \mathrm{~K}$, and the upper gas-cooled leads of a combined HTS and gas cooled lead set. The equivalent $4.4 \mathrm{~K}$ refrigeration can be reduced by a factor of four, when this alternative is used. The second alternative is illustrated in Figure 14 above.

The types of convention Claude cycle refrigerators that are generally available produce more than 30 watts of refrigeration at $4.4 \mathrm{~K}$. Some of the most reliable machines produce refrigeration in the 70 to 100 watt range. If there is several superconducting insertion devices on the light source ring, the use of a conventional refrigerator may be the economic choice. If the insertion device is part of a storage ring that uses superconducting magnets and RF cavities, the refrigerator that is used to cool the rest of the light source should be used to cool the insertion devices as well. 


\section{VIBRATION ISOLATION AND CONNECTION OF COOLING TO THE LOAD}

The connection of the cooler to the load being cooled is often tied to isolating the insertion device from the vibration that is present in the cold head of the cryogenic cooler used to cool the device. The cold of the GM machines experience accelerations of the order of $0.1 \mathrm{~g}$. The vibration forces produced by these machines may be as high as $15 \mathrm{~N}$. The pulse tube coolers have vibration accelerations of the order of $0.001 \mathrm{~g}$, so the vibration forces produced by these machine are about $0.1 \mathrm{~N}$. The cold mass of insertion device magnets varies as the cold gap squared. For most insertion magnets of interest the cold mass is between 100 and $1200 \mathrm{~kg}$. The magnet cold mass alone may not be enough to prevent the cold head accelerations from producing motions in the magnet that will have a bad affect on the quality of the light coming from the insertion device. In most cases additional vibration isolation is required between the cold head of the cooler and the insertion device.

The first element of effective vibration isolation is having stiff cold mass supports. The primary (lowest order) resonant frequency of the cold mass support should be higher than the vibration frequency of the cooler cold head. The second element of effective vibration isolation is to have damping between the cooler and the magnet vacuum vessel. The third element of effective vibration isolation is to have a flexible heat-carrying member between the cooler cold head and the load being cooled. The resonant frequency of the connection between the cold head and the load should be lower than the cold head vibration frequency.

The major problem with vibration isolation with a flexible member is the temperature drop between the insertion device being cooled and the cold head. The temperature drop problem is further complicated by heat leaks down the leads that go from room temperature (or the first stage temperature of the cooler when a two stage cooler is used) to the electronic component being cooled. The literature has a number of papers that describe the issue of how to intercept heat that is entering a device from a higher temperature through its leads.

Two approaches that can be used to isolate the insertion device from cooler vibration are as follows; 1) A flexible metal strap can be used to carry heat from the insertion device and its shields to the cooler cold heads. 2) A flexible heat pipe can be used to connect the cooler cold head to the load. A cryogen free insertion device (with no liquid helium in the device) must use a flexible metallic strap to isolate the cooler from the load. An insertion device that has liquid helium in contact with the magnet may use either a flexible heat pipe on metallic strap for vibration isolation.

\section{Insertion Device Vibration Isolation using a Flexible Copper Strap}

One approach to vibration isolation is to use flexible connectors (multiple thin wires or straps) fabricated from a high thermal conductivity metal (at cryogenic temperatures). A metal that has a high thermal conductivity also has a very low electrical resistivity (at cryogenic temperatures). In general, one wants a material that has a high residual resistance ratio RRR (RRR is the ratio of the electrical resistivity at $273 \mathrm{~K}$ to the electrical resistivity at $4 \mathrm{~K}$ ). There are only few materials that fit the criteria. They are copper, silver, and aluminum. Silver can be eliminated because of its cost and the fact that its low temperature thermal conductivity is not as high as copper. Aluminum is potentially a good candidate because ultra pure aluminum has very high values of RRR and thus a very high thermal 
conductivity at low temperatures. The problem with ultra pure aluminum is how to connect the metal to the cold head and the load without introducing a large temperature drop at the joint. From a practical standpoint copper is probably the only choice that makes real sense. To make aluminum strap with a very low average thermal conductivity from the cold head to the load, more research is probably needed.

Copper that has a low electrical resistivity (high RRR) has a high thermal conductivity. At liquid nitrogen temperature most coppers that have a RRR of 10 or grater will have a thermal conductivity of about $800 \mathrm{~W} \mathrm{~m}^{-1} \mathrm{~K}^{-1}$. At $4.2 \mathrm{~K}$, the resistivity will vary greatly depending on the RRR of the copper. For example, $\mathrm{RRR}=300$ copper will have a thermal conductivity of $1880 \mathrm{~W} \mathrm{~m}^{-1} \mathrm{~K}^{-1}$ at $4.2 \mathrm{~K}$. A copper with $\mathrm{RRR}=5$ will have a thermal conductivity of only $28.8 \mathrm{~W} \mathrm{~m}^{-1} \mathrm{~K}^{-1}$ at $4.2 \mathrm{~K}$.

The temperature drop along a strap is proportional to the heat being carried by the strap, its length, and average thermal conductivity of the copper in the strap. The temperature drop in the strap is inversely proportional to its cross-section area. The temperature drop along a copper strap $\Delta \mathrm{T}_{\mathrm{Cu}}$ can be calculated using the following equation

$$
\Delta T_{C u}=\frac{Q}{A_{C u}} \frac{L_{C u}}{k_{C u}}
$$

where $\mathrm{Q}$ is the heat load from the load to the cold head; $\mathrm{A}_{\mathrm{Cu}}$ is the cross-sectional area of the copper strap; $\mathrm{L}_{\mathrm{Cu}}$ is the length of the strap; and $\mathrm{k}_{\mathrm{Cu}}$ is the thermal conductivity of the strap. Using the Wiedemann Franz law one can estimate the value of $\mathrm{k}_{\mathrm{Cu}}$ if one knows the average temperature $\mathrm{T}$ and the electrical resistivity $\rho_{\mathrm{Cu}}$ of the copper. One should use the following expression to estimate $\mathrm{k}_{\mathrm{Cu}}$;

$$
k_{C u}=\frac{L T}{\rho_{C u}}
$$

$-28-$

where $\mathrm{L}$ is the Lorenz number $\left(\mathrm{L}=2.45 \times 10^{-8} \mathrm{~W} \Omega \mathrm{K}^{-2}\right)$

In general, the total cross-section area of a flexible strap is much smaller than the area of the cooler cold head. For the best vibration isolation the strap must be made as long as one can make it. As a result, the temperature drop along the strap may be larger than is desired. The joints that connect the flexible strap to the load and the cooler cold head add to the temperature drop.

A copper strap must be used to connect the cold head to the insertion device, if the insertion device is cryogen free. This means that the second stage $(4.2 \mathrm{~K})$ cold head must be close to the device in order to keep the temperature drop from the insertion device to the cold head less than $0.3 \mathrm{~K}$. If a tank of liquid helium is connected to the device to provide cooling to the device in the event of a power failure or a cooler failure, the cooler second stage cold head must still be connected directly to the load through the copper strap. Do not connect the cooler cold head through the a copper strap and liquid helium. Liquid helium is a poor conductor of heat. A cryogen free insertion device or a device with extra helium to provide emergency cooling must be surrounded with copper plates so that heat from the device will get to the device end of the flexible copper strap that connects to the cooler cold head. The total temperature drop from the high field part of the insertion device superconductor to the cooler second 
stage must be as low as one can make it (less than $0.3 \mathrm{~K}$ ). The temperature drop from the shields and the tops of the HTS leads can be much higher (of the order of 10 to $20 \mathrm{~K}$ ). The ratio of the heat load into the first stage to the heat load into the second stage is about equal to the ratio of the allowable temperature drops for both stages. Therefore, on both stages, the distances from the cold head to the load are relatively short. One must do careful engineering in order to have the $\Delta \mathrm{T}$ from the load to the cold head in the correct range for both stages of the cooler. Figure 15 illustrates how the Superbend dipole cold mass is connected to a Sumitomo RDK-415D cooler.

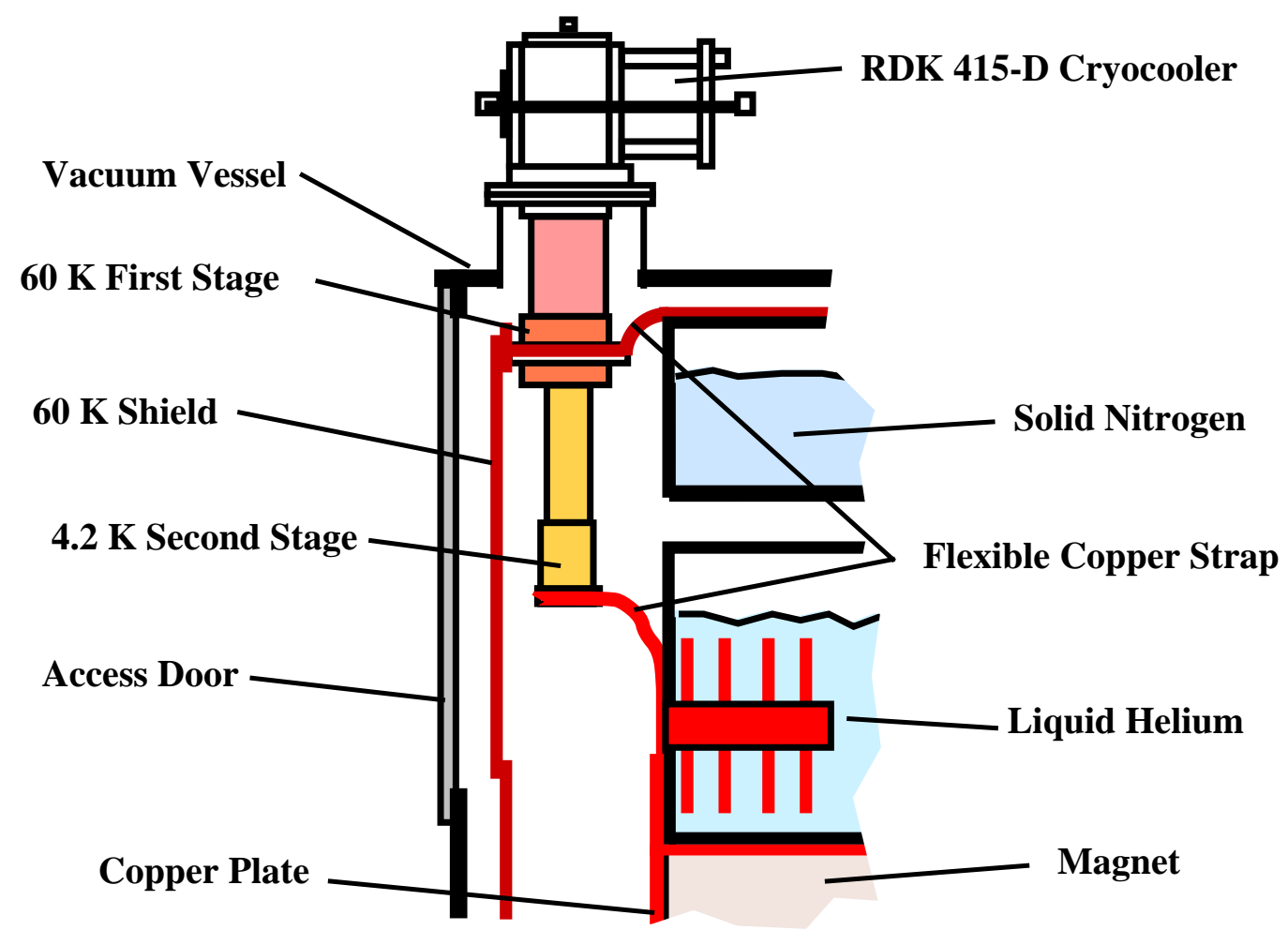

Figure 15. Connection of the Superbend Dipole Cold Mass to Both Stages of a Sumitomo RDK-415D Cooler

Superbend shown in Figure 15 has a copper strap that is about 0.14-meters long that connects the second stage of the cooler to the magnet and its helium tank. The cross-section area of this strap is about $0.0005 \mathrm{~m}^{2}$. With a heat load of $.1 .4 \mathrm{~W}$ flowing through into the cold heat (at $4.15 \mathrm{~K}$ ), the $\Delta \mathrm{T}$ from the load to the cold head is about $0.45 \mathrm{~K}$. This temperature drop is marginally OK for the Superbend magnet, but it would be unacceptable for a high performance insertion device fabricated from niobium titanium superconductor. The strap between the first stage and the nitrogen tank is about 0.1-meters long and it has a cross-section area of $0.00045 \mathrm{~m}^{2}$. The $\Delta \mathrm{T}$ in this strap is $13 \mathrm{~K}$ when $40 \mathrm{~W}$ is carried from the nitrogen tank to the first stage of the cooler. The $13 \mathrm{~K}$ temperature drop at $55 \mathrm{~K}$ is fine for this application and it would also be fine for an insertion device as well.

A copper strap conducts heat in both directions. When the cooler is shut off due to a power failure or a cooler failure, heat will flow from the cooler to the liquid nitrogen and the liquid helium tanks. 
When the first stage of the Sumitomo RDK-415D cooler is kept cold by liquid nitrogen in the nitrogen tank (the first stage cold head is at 90 to $95 \mathrm{~K}$ ), the heat leak from the second stage of the cooler into the liquid helium tank is about $2.0 \mathrm{~W}$ [18]. Most of the temperature drop from the first stage cold head to the second stage cold head is in the second stage cold head itself. With the upper part of the Sumitomo RDK-415D machine at $300 \mathrm{~K}$, the estimated heat leak into the nitrogen tank through the first stage cold head is 50 to $60 \mathrm{~W}$. Most of the temperature drop from the $300 \mathrm{~K}$ parts of the cooler to the first stage cold head at 90 to $95 \mathrm{~K}$ is due to heat flow in the cooler.

We know from measurements on Superbend that a magnet can operate on liquid cryogens even with a Sumitomo RDK-415D cooler attached to the load and shut off. Since the temperature drop in both heads of the cooler is dominated by temperature drops within the cooler, improving the heat transfer in the strap for a given $\Delta \mathrm{T}$ is not going to make much difference to the cryogen boil off when the cooler is shut off. One might expect a pulse tube cooler to behave in a way that is similar to the Sumitomo GM cooler, but LBNL has not measured how much heat leak would come into a liquid helium and liquid nitrogen baths from a pulse tube cooler that is shut off.

\section{Insertion Device Vibration Isolation and Connection to the Cold Head through a Heat pipe}

An approach to improving the stability of a high current density insertion device magnet is to immerse the device in liquid helium. The temperature of the magnet is very close to the temperature of the liquid helium the magnet is operating in. Liquid helium is both a blessing and a curse for devices being cooled using small cryo-coolers. LBNL ha s learned from experience that one can not just cool a pot of liquid helium with a cryo-cooler and expect the temperature drop from the magnet (the liquid helium) to be low. A paper concerning the LBNL experience on the Venus magnet system will be reported at the 2003 Cryogenic Engineering Conference [22].

Clyde Taylor of LBNL found that the key to connecting the cooler cold head with the load immersed in a cryogenic fluid is creating a condenser that causes the cryogen that the cools the load to circulate [22]. Simply immersing the cold head of the cooler into a bath of liquid cryogen does not work if one wants a low temperature drop between the source of the heat and the cold head to be low. The difference between making the connection from the cold head to the load correctly and not making the connection correctly is a factor of 10 to 30 (depending on the case) difference in the effective heat transfer coefficient between the cooled load and the cryo-cooler cold head. Furthermore, excellent vibration isolation can be achieved because the distance from the load to the cold head can be increased and the elements connecting the two can be flexible within the pressure constraints of the cooling circuit. The device that Clyde Taylor proposed is a gravity separated heat pipe. Heat pipes, if properly designed, can have very high heat transfer coefficients and they can transfer heat over long distances. Figure 16 shows a schematic of a heat pipe system that Clyde Taylor built for the Venus magnet system for transmitting heat from the magnet to the cold head. The heat pipe shown in Figure 16 can be used with liquid helium or liquid nitrogen. A liquid helium heat pipe can operate over a temperature range from 2.5 to $5.0 \mathrm{~K}$. A liquid nitrogen heat pipe can be used to transmit heat over a range of temperature between $65 \mathrm{~K}$ and $100 \mathrm{~K}$. Heat pipes can be used for other cryogenic fluids as well [23]. 


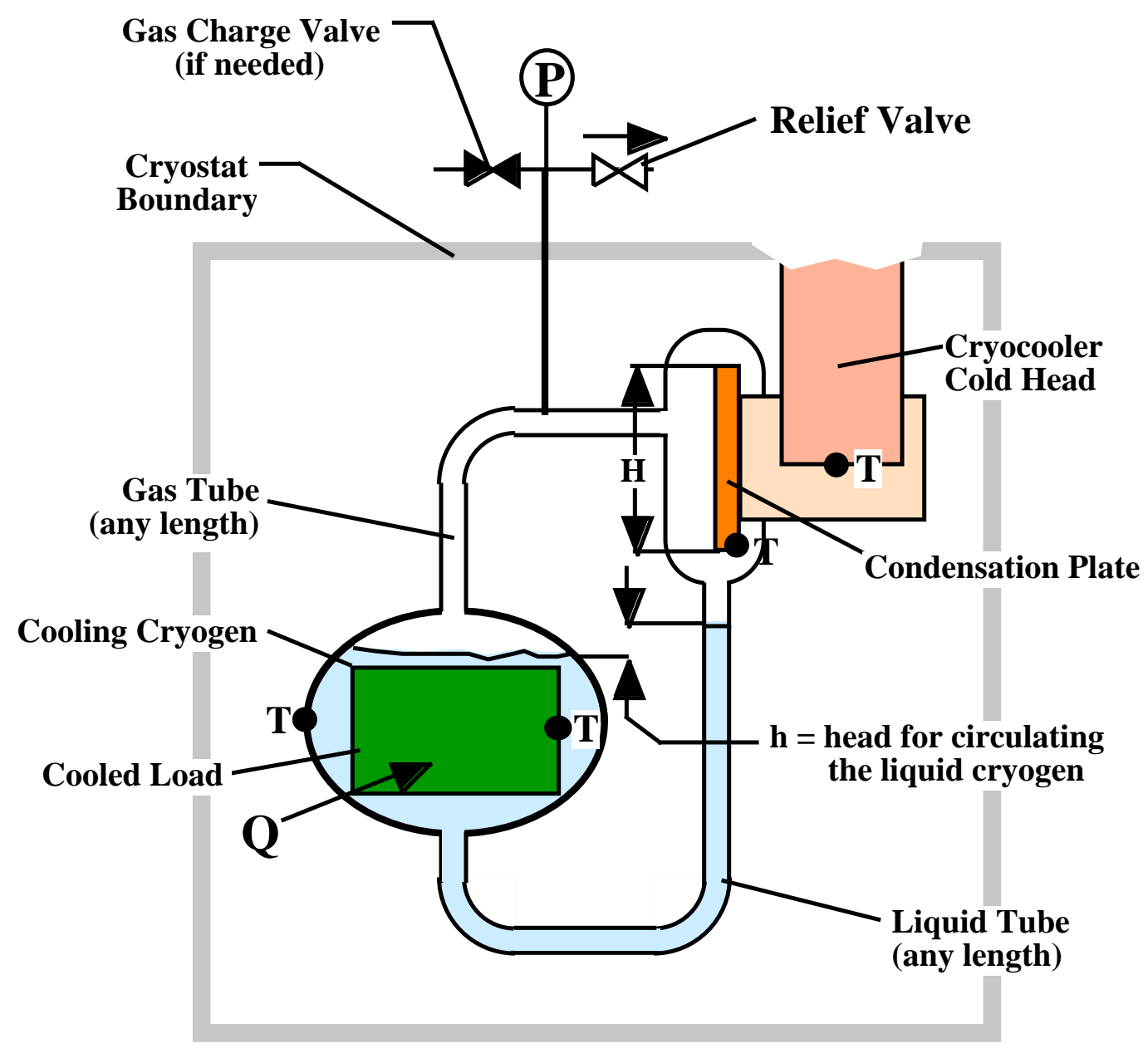

Figure 16. A Schematic Representation of a Heat Pipe Heat Transfer System for a Magnet Immersed in a Liquid Cryogen (Note: the system shown is an open cycle system. Closed heat pipes can also be designed.)

Figure 16 shows a schematic representation of a cooler with a condenser attached to the cold head. Figure 16 is nothing but a schematic representation of a heat pipe that uses gravity to separate the liquid and gas phases. The boiling surface for the heat pipe is the cooled load that is immersed in liquid helium. For the heat pipe to transmit heat from the insertion device magnet being cooled to the cooler cold head, the hotter surface must be in liquid cryogen that is physically below the condenser surface, which is in gas. The temperature drop $\Delta \mathrm{T}$ from the load being cooled and the cooler cold head is a function of the heat transfer per unit area $\mathrm{Q} / \mathrm{A}$ of both the boiling and condensing surfaces, the surface orientation (both surfaces), the height of the condensing surface $\mathrm{H}$, and the fluid properties. In order for a gravity driven heat pipe shown in Figure 16 to function properly, the following must be true: 1) the condensing surface must be oriented so that the fluid flows off the surface in a thin layer; 2) The condensing surface must not be flooded with liquid; 3) the boiling surface should face up or to the side (if both sides of a device are cooled); and 4) the pipes between the boiling and condensing surfaces must be large enough to carry the flow with a low pressure drop. 
The potential advantages of using a heat pipe to carry heat from the device being cooled to the cooler cold head are as follows: 1) Heat can be transferred from the device to the cold head with a small total temperature drop $\Delta \mathrm{T}$. This advantage is particularly useful for devices that generate a lot of heat (say over 1 watt) at liquid helium temperature. (The use of a heat pipe in a large device (about $3.1 \mathrm{~W}$ ) at LBNL reduced the $\Delta \mathrm{T}$ from the device to the cold head from $450 \mathrm{mK}$ to $30 \mathrm{mK}$ [22].) 2) Coolers that produce temperatures in the $4 \mathrm{~K}$ range produce temperature oscillations on the cold head in the range of 0.25 to $0.3 \mathrm{~K}$ peak to peak. For a superconducting magnet, this is not a large concern because the mass of the magnet helps filter out the temperature variation. A heat pipe will filter these temperature oscillations almost completely. 3) A heat pipe can carry heat from the device to the cold head over long distances, because the $\Delta \mathrm{T}$ from the device to the cold head is not a function of the distance between the heat load and the cold head, to first order. This statement applies for a liquid nitrogen heat pipe as well as a liquid helium heat pipe. 4) Because heat can be transferred over long distances almost without penalty, the cooler can be located so that changing out coolers can be done with ease. 5) A properly designed heat pipe, (particularly one that carries heat over a long distance) will act as a thermal diode. Heat transfer from the device being cooled to the cold head will be very good as long as the cold head is colder than the device. If the cold head is warmer than the device, the heat transfer going from the cold head to the device will be poor. This effect can be dramatic even at liquid nitrogen temperature. The use of a heat pipe as a thermal diode is not a new idea. NASA was doing this over 35 years ago.

The primary disadvantage of using a heat pipe to connect the device to the cooler cold head is the extra complication involved with fabricating the heat pipe system. The pipes must be long and coiled ore they must be bellows pipes, if good vibration isolation is desired. The gravity separation heat pipe shown in Figure 1 will not operate in a zero g environment (there are heat pipes that will) or on devices where the direction of gravity changes more than 30 degrees.

In Figure 16, the insertion device magnet is immersed in a helium bath at its saturation temperature $\mathrm{T}_{\mathrm{s}}$. The magnet boils the helium. The helium gas flows to the condenser through the tube marked as the gas tube. Liquid helium drips off the condenser surface and returns to the liquid bath through the tube marked liquid tube. As long as the condenser is physically above the surface of the liquid cryogen (with gravity acting in the downward direction), the cryogen will circulate from the heat source to the condenser as a gas and from the condenser back to the heat source as a liquid. The circulating mass flow $\mathrm{M}=\mathrm{Q} / \mathrm{h}_{\mathrm{fg}}$ where $\mathrm{Q}$ is the heat load and $\mathrm{h}_{\mathrm{fg}}$ is the heat of vaporization of the fluid at its saturation temperature.

The heat transfer coefficient for a condenser of height $\mathrm{H}$ for a temperature drop across the condenser $\Delta \mathrm{T}_{\mathrm{c}}$ will take the following general form [24];

$$
h_{c}=0.94\left[\frac{\rho_{l}^{2} g h_{f g} k_{l}^{3}}{\mu_{l} H \Delta T_{c}}\right]^{0.25}
$$

where $\rho_{1}$ is the density of the liquid cryogen [25]; $g$ is the acceleration of gravity; $k_{1}$ is the thermal conductivity of the saturated liquid; and $\mu_{1}$ is the viscosity of the liquid. $\mathrm{H}, \mathrm{h}_{\mathrm{fg}}$ and $\Delta \mathrm{T}_{\mathrm{c}}$ are previously 
defined. From Equation 1 one can define the heat transfer area per unit area in the condenser $(\mathrm{Q} / \mathrm{A})_{\mathrm{c}}$;

$$
\left(\frac{Q}{A}\right)_{c}=\left[\frac{\rho_{l}^{2} g h_{f_{g}} k_{l}^{3}}{\mu_{l} H}\right]^{0.25} \Delta T_{c}^{0.75}
$$

The temperature drop across the condenser $\Delta \mathrm{T}_{\mathrm{c}}$ can be estimated using the following expression;

$$
\Delta T_{c}=\left[\left(\frac{Q}{A}\right)_{c}\left(\frac{\mu_{l} H}{\rho_{l}^{2} g h_{f g} k_{l}^{3}}\right)^{0.25}\right]^{\frac{4}{3}}
$$

From Equation 3, it is clear that the temperature drop on the condensing surface is a function of the heat transfer per unit area on that surface. Extending the surface using will reduce the $\Delta \mathrm{T}_{\mathrm{c}}$ provided the fins are vertical so the condensed liquid can flow off of them.

The peak nucleate boiling heat flux per unit area $[\mathrm{Q} / \mathrm{A}]_{\mathrm{np}}$ and the peak temperature difference between the hot surface and the bath $\Delta \mathrm{T}_{\mathrm{np}}$ during nucleate boiling can be estimated using the following expressions [26];

$$
\begin{aligned}
& {\left[\frac{Q}{A}\right]_{n p}=0.16 h_{f g} \rho_{v}^{0.5}\left[\sigma g\left(\rho_{l}-\rho_{v}\right)\right]^{0.25}} \\
& \Delta T_{n p}=0.5\left[1-\frac{T_{s}}{T_{c}}\right]^{1.5} T_{c}
\end{aligned}
$$

where $\rho_{1}$ is the density of the saturated liquid $\rho_{v}$ is the density of the saturated vapor; $\sigma$ is the surface tension; $\mathrm{T}_{\mathrm{s}}$ is the saturation temperature of the fluid; and $\mathrm{T}_{\mathrm{c}}$ is the critical temperature of the fluid. Equation 5 is good over a range of $0.56<\mathrm{T}_{\mathrm{s}} / \mathrm{T}_{\mathrm{c}}<1$. For $\mathrm{Q} / \mathrm{A}<(\mathrm{Q} / \mathrm{A})_{\mathrm{np}}, \mathrm{Q} / \mathrm{A}$ is proportional to $\Delta \mathrm{T}^{2.5}$.

One would never design a system to operate at the peak nucleate boiling point. It would be prudent to design the boiling system so that boiling occurs at a heat flux per unit area $(\mathrm{Q} / \mathrm{A})_{\mathrm{b}}$ well below the minimum film boiling heat flux $(\mathrm{Q} / \mathrm{A})_{\mathrm{f}}$. The boiling temperature drop $\Delta \mathrm{T}_{\mathrm{b}}$ for the boiling side) can be expressed as follows;

$$
\Delta T_{b}=\left[\frac{(Q / A)_{b}}{(Q / A)_{n p}}\right]^{0.4} \Delta T_{n p}
$$

From Equation 6, it is clear that the $\Delta \mathrm{T}_{\mathrm{b}}$ on the boiling surface is a function of $(\mathrm{Q} / \mathrm{A})_{\mathrm{b}}$. If wants to reduce the value of $\Delta \mathrm{T}_{\mathrm{b}}$, one must increase the boiling area. Extending the surface is not an issue for cooling an insertion device magnet, because the coil surface area is large and the heat to be transferred from the magnet is relatively small. For the most part $\Delta \mathrm{T}_{\mathrm{b}}$ will always be less than $\Delta \mathrm{T}_{\mathrm{c}}$. As a matter of reference, the minimum film boiling heat flux for liquid helium $(\mathrm{Q} / \mathrm{A})_{\mathrm{f}}=2000 \mathrm{~W} \mathrm{~m}^{-2}$; and for liquid 
nitrogen $(\mathrm{Q} / \mathrm{A})_{\mathrm{f}}=8000 \mathrm{~W} \mathrm{~m}^{-2}$ [27] [28]. As with $\Delta \mathrm{T}_{\mathrm{c}}$, the value of $\Delta \mathrm{T}_{\mathrm{b}}$ can be set by controlling the heat transfer per unit area of the boiling surface. The total temperature drop from the magnet to the cold head from Equations 31 and $33 \Delta \mathrm{T}_{\mathrm{T}}$ is;

$$
\Delta T_{T}=\Delta T_{c}+\Delta T_{b}
$$

The effective heat transfer coefficient for the combined boiler and condenser can be estimated using the following expression;

$$
h_{b c}=\frac{Q}{A_{t} \Delta T_{T}}
$$

where $\mathrm{Q}$ is the heat generated at the load; and $\mathrm{A}_{\mathrm{t}}$ is the cross-section area of the two tubes. The parameters of helium and nitrogen are shown in Table 5. From these parameters one can calculate the $\Delta \mathrm{T}$ and heat transfer coefficients for both helium and nitrogen heat pipes.

Table 5. The Physical Properties of Liquid and Gaseous Helium and Nitrogen [25]

\section{Fluid Parameter}

Triple Point Temperature (K)

Triple Point Pressure (atm)

Critical Temperature (K)

Critical Pressure (atm)

Boiling Temperature at 1 atm $\mathrm{T}_{\mathrm{b}}(\mathrm{K})$

Heat of Vaporization at $\mathrm{T}_{\mathrm{b}}\left(\mathrm{J} \mathrm{kg}^{-1}\right)$

Saturated Liquid Density at $\mathrm{T}_{\mathrm{b}}\left(\mathrm{kg} \mathrm{m}^{-3}\right)$

Saturated Vapor Density at $\mathrm{T}_{\mathrm{b}}\left(\mathrm{kg} \mathrm{m}^{-3}\right)$

$\mathrm{C}_{\mathrm{p}}$ Saturated Liquid at $\mathrm{T}_{\mathrm{B}}\left(\mathrm{J} \mathrm{kg}^{-1} \mathrm{~K}^{-1}\right)$

$\mathrm{C}_{\mathrm{p}}$ Saturated Vapor at $\mathrm{T}_{\mathrm{b}}\left(\mathrm{J} \mathrm{kg}^{-1} \mathrm{~K}^{-1}\right)$

Thermal Conductivity of Liquid at $\mathrm{T}_{\mathrm{b}}\left(\mathrm{W} \mathrm{m}^{-1} \mathrm{~K}^{-1}\right)$

Thermal Conductivity of Vapor at $\mathrm{T}_{\mathrm{b}}\left(\mathrm{W} \mathrm{m}^{-1} \mathrm{~K}^{-1}\right)$

Viscosity of Saturated Liquid at $\mathrm{T}_{\mathrm{b}}\left(\mathrm{kg} \mathrm{m}^{-1} \mathrm{~s}^{-1}\right)$

Viscosity of Saturated Vapor at $\mathrm{T}_{\mathrm{b}}\left(\mathrm{kg} \mathrm{m}^{-1} \mathrm{~s}^{-1}\right)$

\section{Cryogenic Fluid}

$\begin{array}{cc}\text { Helium } & \text { Nitrogen } \\ 2.17 & 63.1 \\ 0.051 & 0.128 \\ 5.19 & 126.1 \\ 2.21 & 33.8 \\ 4.22 & 77.3 \\ 20900 & 199700 \\ 125 & 808 \\ 16.1 & 4.59 \\ \sim 2500 & \sim 2040 \\ 5200 & 1040 \\ 0.027 & 0.140 \\ 0.011 & \sim 0.0075 \\ 3.53 \times 10^{-6} & 1.58 \times 10^{-4} \\ 0.91 \times 10^{-6} & \sim 5.9 \times 10^{-6}\end{array}$

When one looks at Equation 35, one sees that the heat transfer coefficient for the condenser and boiler system (the heat pipe) is independent of the distance separating them. The $\Delta \mathrm{T}$ from the boiling surface to the condenser surface is also independent of distance between the two surfaces (See Equation 34). When one looks at Equation 27, one sees the $\Delta \mathrm{T}$ along a copper strap is proportional to its length. This means that a properly designed heat pipe can be designed so that the heat load can be quite far from 
the cooler cold head and condenser. In order to increase the distance between the boiling and condensing surfaces, one must size the pipe so that the pressure drop does not change as one increases the length between the two surfaces. In general, the flow pipe diameter scales with the distance between the boiling and condensing surfaces to the 0.21 power (assuming a smooth pipe, for a bellows pipe the scaling power should be about 0.25 ).

Table 6 compares the performance of a well-designed heat pipe with a pure copper strap as a means to transfer heat from an insertion device magnet to the cold head of a cooler. This comparison is done at 4.2 K, and $77 \mathrm{~K}$. The fluids used in the heat pipe are helium and nitrogen respectively. The heat transferred per unit area $(\mathrm{Q} / \mathrm{A})$ and the temperature drop $\Delta \mathrm{T}$ are compared for the two methods over distances of $150 \mathrm{~mm}$ and $1000 \mathrm{~mm}$. For the copper strap Q/A is defined as the heat transferred divided by the strap cross-section area. The Q/A used for the heat pipe is based the heat transferred divided by the liquid cross-section area of the liquid tube plus the gas cross-section area of the gas tube. The value of Q/A used for the helium tubes was based on the tubes used for the experiment [21]. The theoretical Q/A for a helium heat pipe may be higher than that used in Table 6.

Table 6. A Comparison of the Heat Transfer of a Copper Strap and a Heat Pipe at $4.2 \mathrm{~K}$ and $77.3 \mathrm{~K}$

$\begin{array}{ccccc}\text { Heat Transfer Method } & \mathrm{T} & \mathrm{Q} / \mathrm{A} & \text { Length } & \Delta \mathrm{T} \\ & (\mathrm{K}) & \left(\mathrm{W} \mathrm{m}^{-2}\right) & (\mathrm{m}) & (\mathrm{K}) \\ \text { Copper Strap } & 4.2 & 2600 & 0.15 & 0.48 \\ \text { Copper Strap } & 4.2 & 2600 & 1.00 & 3.18 \\ \text { Helium Heat Pipe } & 4.2 & 25000 & 0.15 & 0.05 \\ \text { Helium Heat Pipe } & 4.2 & 16800 & 1.00 & 0.05 \\ \text { Copper Strap } & 77.3 & 13000 & 0.15 & 2.45 \\ \text { Copper Strap } & 77.3 & 13000 & 1.00 & 16.25 \\ \text { Nitrogen Heat Pipe } & 77.3 & 64000 & 0.15 & 1.04 \\ \text { Nitrogen Heat Pipe } & 77.3 & 43100 & 1.00 & 1.04\end{array}$

The calculation for the heat pipe are based on the following values heat transfer per unit area for the condensing surface and the boiling surface: for helium, $(\mathrm{Q} / \mathrm{A})_{\mathrm{c}}=40 \mathrm{~W} \mathrm{~m}^{-2}$ and $(\mathrm{Q} / \mathrm{A})_{\mathrm{b}}=2 \mathrm{~W} \mathrm{~m}^{-2}$; and for nitrogen, $(\mathrm{Q} / \mathrm{A})_{\mathrm{c}}=2000 \mathrm{~W} \mathrm{~m}^{-2}$ and $(\mathrm{Q} / \mathrm{A})_{\mathrm{b}}=200 \mathrm{~W} \mathrm{~m}^{-2}$. The residual resistance ratio $\mathrm{RRR}$ for the copper strap is assumed to be 130 (the same as the strap used for Superbend). If the strap RRR is higher, the $\Delta \mathrm{T}$ will be lower at $4 \mathrm{~K}$. On the other hand, if the copper is cold worked so that the RRR goes down, the $\Delta \mathrm{T}$ at $4 \mathrm{~K}$ will be higher than shown in Table 6 . The RRR of the copper in the strap has a small effect on strap heat transfer performance at $77 \mathrm{~K}$. The $\Delta \mathrm{T}$ for the copper strap cases can be adjusted by changing the Q/A of the copper. At $4.2 \mathrm{~K}$, one must reduce Q/A in the $150 \mathrm{~mm}$ long copper strap by an order of magnitude in order to get any where near the same performance as the heat pipe gets over any distance up to a meter. There is a strong incentive to use a heat pipe to connect the second stage of the cooler to the liquid bath around the insertion magnet. 


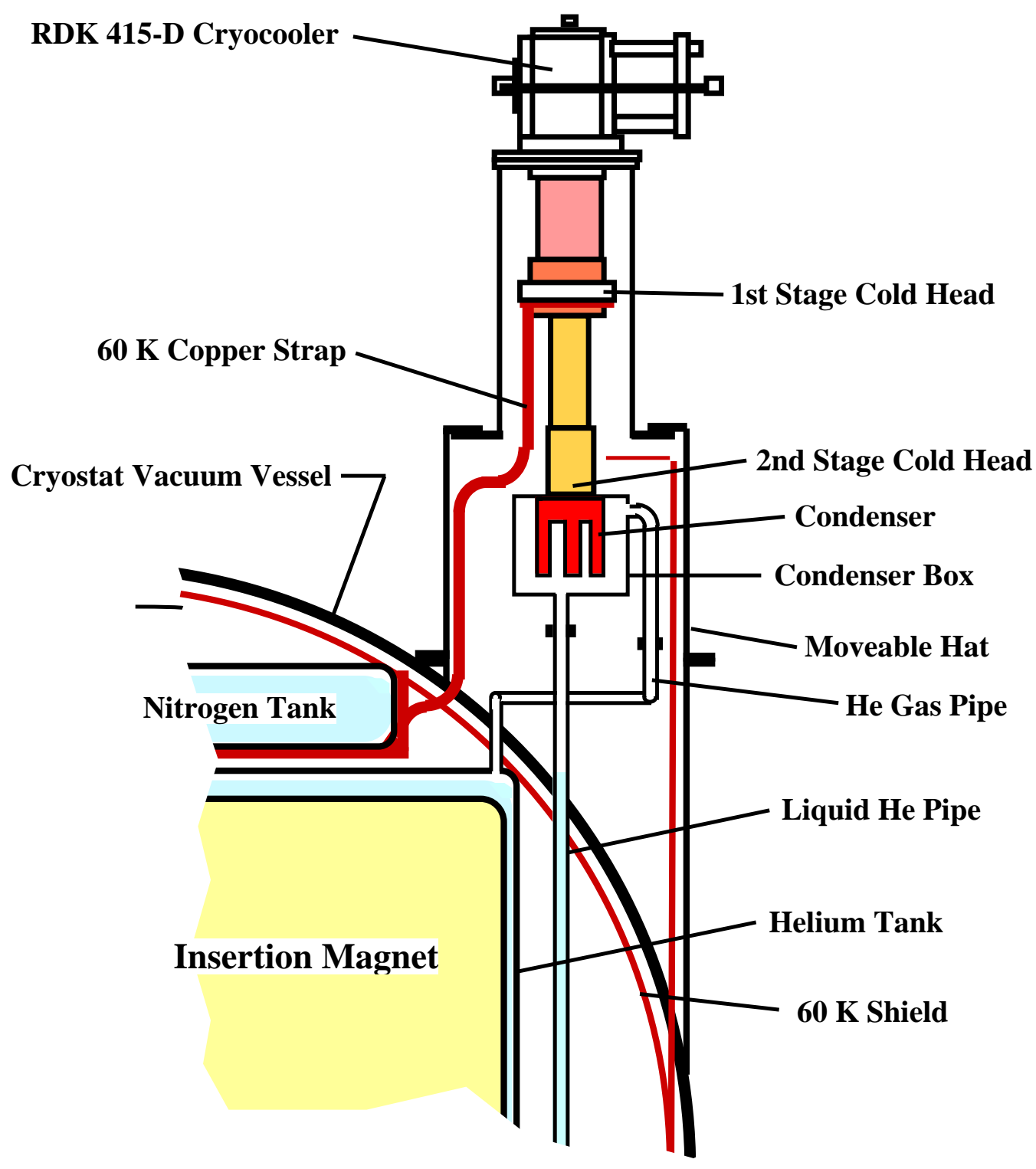

Figure 17. A Representation of how a Small Cryo-cooler can be attached to an Insertion Magnet using a Helium Heat Pipe (Note: the nitrogen temperature stage of the cooler is attached to the nitrogen tank using a copper strap.)

Figure 17 shows how one can mount the cryo-cooler and a helium heat pipe system to an insertion magnet. The cooler shown in Figure 17 could be one of up to three coolers that are attached to an insertion magnet that is up to 2-meters long. A copper strap is shown attaching the nitrogen tank to the first stage of the cooler. The cross-sectional area of the strap can be sized so that the $\Delta \mathrm{T}$ between the first stage cold head and the liquid nitrogen tank is kept below about $10 \mathrm{~K}$. If the temperature in the nitrogen tank is below the triple point temperature $(63.1 \mathrm{~K})$, the nitrogen will be a sold.

A nitrogen heat pipe can be used to carry heat from the liquid nitrogen tank to the cold head, but the nitrogen condenser attached to the first stage cold head must never be allowed to go below the triple point temperature for nitrogen. If it does, the nitrogen will freeze on the condenser and the heat pipe 
will cease to carry heat from the nitrogen tank to the cold head. If one uses a nitrogen heat pipe to carry heat from the nitrogen tank to the cooler first stage, a heater must be mounted on the cooler first stage so that its temperature is kept above $65 \mathrm{~K}$.

The system as shown in Figure 17 will allow for a cooler to be shut off when say three coolers are cooling the load. With the second stage cold head warmer than the helium bath, heat will not flow from the magnet cryostat to the cold head. If the heat pipe plumbing is designed to eliminate the thermal acoustic oscillation heat transfer from $4 \mathrm{~K}$ to the condenser (a cotton cord can be run up the tube), the heat flow from the helium tank to the condenser box will be very poor. Since the helium in the pipes will be stratified, the heat transferred from the condenser box to the helium bath will be by conduction down the pipe walls. The heat transfer from the condenser box to the helium bath can be estimated using the following expression;

$$
Q=\frac{\Gamma A_{c t}}{L_{t}}
$$

where $A_{c t}$ is the cross-sectional area of the tubes; $L_{t}$ is the length of the tube; and $\Gamma$ is the thermal conductivity integral. The thermal conductivity integral $\Gamma$ from temperature $T_{1}$ to temperature $T_{2}$ can be expressed as follows;

$$
\Gamma=\int_{T_{1}}^{T_{2}} k(T) d T=\frac{L\left(T_{2}^{2}-T_{1}^{2}\right)}{\left(\rho\left(T_{2}\right)+\rho\left(T_{1}\right)\right)}
$$

where $\mathrm{L}$ is the Lorenz number $\left(\mathrm{L}=2.45 \times 10^{-8} \mathrm{~W} \Omega^{-1} \mathrm{~K}^{-2}\right) ; \rho\left(\mathrm{T}_{1}\right)$ is the electrical resistivity of the tube material at temperature $\mathrm{T}_{1}$; and $) ; \rho\left(\mathrm{T}_{2}\right)$ is the electrical resistivity of the tube material at temperature $\mathrm{T}_{2}$. If one makes the flexible tubes in the heat pipe from 304 stainless steel, the electrical resistivity $\rho$ through out the temperature range from $4 \mathrm{~K}$ to $400 \mathrm{~K}$ is about $4 \times 10^{-7} \Omega \mathrm{m}$. Thus the thermal conductivity integral has the approximate following values; from $4 \mathrm{~K}$ to $77 \mathrm{~K}, \Gamma=196 \mathrm{~W} \mathrm{~m}^{-1}$ and from 4 to $300 \mathrm{~K}, \Gamma=196 \mathrm{~W} \mathrm{~m}^{-1}$.

If the tubes shown in Figure 17 are stainless steel tubes $12.7 \mathrm{~mm}$ in diameter, with a $0.5 \mathrm{~mm}$ wall and are $0.5 \mathrm{~m}$ long, the heat leak into the helium vessel down the tubes is $0.016 \mathrm{~W}$ when the condenser box is at $77 \mathrm{~K}$. When the condenser box is at $300 \mathrm{~K}$ (the case when both the helium and nitrogen circuits are connected using heat pipes and the cooler is off), the heat leak into the helium is $0.22 \mathrm{~W}$.

The refrigeration to the insertion device must be monitored by measuring the temperatures at the cooler cold head and on the magnet. Liquid level should not be used as a method for monitoring or controlling the refrigeration. The expansivity of liquid helium at $4.4 \mathrm{~K}$ is $0.209 \mathrm{~K}^{-1}$, which means that when the helium warms from $3.9 \mathrm{~K}$ to $4.9 \mathrm{~K}$, it occupies 20 percent more volume. The temperature of the cold head tells one the amount of heat that is entering the cooler through the cold head. The $\Delta \mathrm{T}$ between the magnet and the cold will be proportional to the heat being generated in the magnet. The temperature of the liquid helium in the magnet cryostat will be between the magnet temperature and the cold head temperature. The pressure of the helium in the cryostat is the helium saturation pressure. 


\section{Cooling Connection to the Insertion Device from a Conventional Refrigerator}

A conventional refrigerator with a J-T circuit may be connected to the insertion magnet cryostat as shown in Figures 13 and 14. Two-phase helium enters the cryostat through the J-T valve. The liquid and gas phases are separated in the helium can. The helium gas returned to the refrigerator is as at the saturation temperature of the helium in cryostat. All of the helium that is in the magnet cryostat will boil in the event of a magnet quench. Since the stored energy of insertion devices tends to be low (less than $50 \mathrm{~kJ}$ ), this is not a problem that is worth considering. An alternative is to run the magnet with little or no helium in contact with the magnet coils. This alternative is shown in Figure 18.

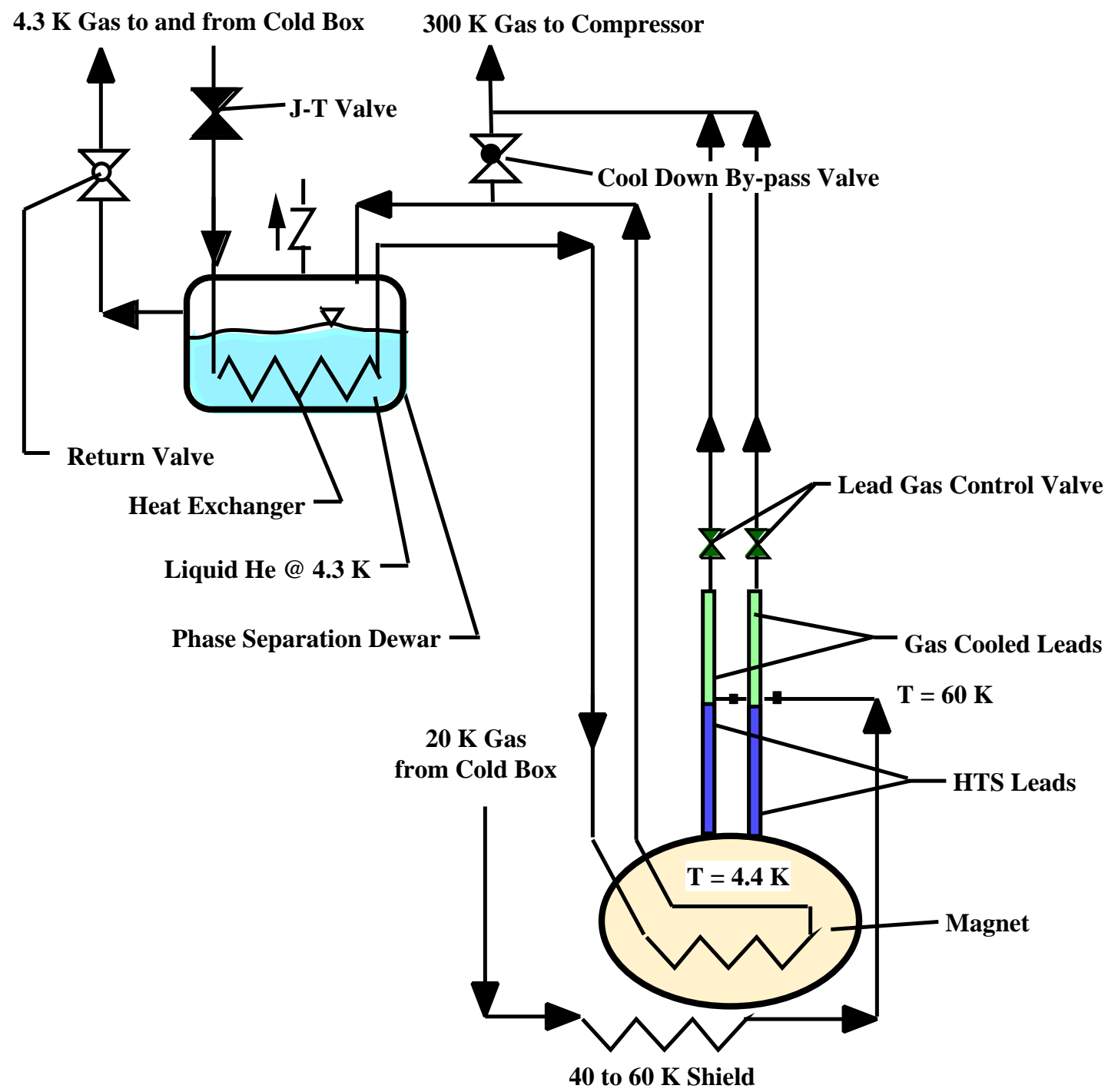

Figure 18. A Schematic of a Forced Two-Phase Cooling System for a Insertion Magnet using a Conventional Refrigerator (Note: During a cool down close the return valve and open the cool down by-pass valve.)

The approach shown in Figure 18 is cooling the magnet using forced two-phase helium in tubes (there is very little liquid helium near the magnet) [29]. In this case liquid helium is stored in a separate 
storage cryostat. The gas part of the two-phase flow from the J-T valve is condensed in a heat exchanger within this storage cryostat. The helium leaves the heat exchanger as a sub-cooled liquid. As the helium goes through the magnet it is warmed up and evaporated. The role of the storage dewar and heat exchanger is move the position of the two-phase helium flowing through the tubes in the magnet from the gas side of the two-phase dome to the liquid side of the two-phase dome [29] [30]. While force two phase cooling has been widely used for detector magnets, it has some disadvantages because the magnet typically runs about $0.1 \mathrm{~K}$ warmer than the helium in the control dewar. One should expect the operating temperature of an insertion magnet run off of a conventional refrigerator to be about $4.35 \mathrm{~K}$. If the insertion magnet is cooled using force two-phase helium (as shown in Figure 18), its operating temperature would be in the range from 4.4 to $4.5 \mathrm{~K}$

A conventional refrigerator can be some distance (even over 100 meters) from the insertion device being cooled. The heat leaks into the transfer lines must be accounted for. Since the refrigerator cold box can be some distance from the insertion magnet, cold box vibration should not be an issue when an insertion device is run off of a conventional refrigerator. The compressors for a conventional refrigerator can be 100 meters or more from the cold box. Modern screw compressors are a relatively small source of vibration that might be seen by a light source. Conventional refrigerator vibration is a relatively straightforward issue to deal with, so it should not be a problem for insertion devices.

\section{COOL DOWN OF INSERTION DEVICES}

This section will discuss cooling down insertion device magnets with liquid cryogenic fluids (nitrogen and helium), with the small coolers used to keep the devices cold over a long period of time, or with conventional larger refrigerators. For the devices discussed in this report, design the $4.2 \mathrm{~K}$ heat leak into the cryostat is relatively small (from about 1 watt to perhaps 45 watts for a long device with a $4.2 \mathrm{~K}$ bore). The magnet system cold mass for these devices will range from $150 \mathrm{~kg}$ for a small device to about $1500 \mathrm{~kg}$ for a large device (including the cryogenic tanks).

The size of the refrigerator used to cool the insertion device is determined by the heat flow into the system at $4 \mathrm{~K}$ and the total current of all of the leads bringing current into the device. In general, the device either has a low enough heat leak and total current so that it can be kept cold with from one to three $1.5 \mathrm{~W}$ coolers or it is a device will be cooled using a conventional refrigerator. With the low heat leak devices, one either cools them down using liquid nitrogen and helium or one uses the coolers to cool them down. The devices that are cooled using conventional refrigerators would be cooled down using the refrigerator that is supposed to keep the device cold over a period of time.

\section{Insertion Device Cool Down using Liquid Nitrogen and Liquid Helium}

Since most of the insertion devices discussed here will have reservoirs for liquid helium and liquid nitrogen, the cool down of these devices is relatively straight forward using liquid nitrogen and liquid helium. The mass of liquid nitrogen and liquid helium needed to cool the device down depends on the total cold mass of the device and the materials that make up the device. In general, one should cool the 
device to 80 to $100 \mathrm{~K}$ using liquid nitrogen, and then liquid helium is used to cool the device down to 4.2 K. There must be no liquid nitrogen in the liquid helium tank, when liquid helium is used for cooling the device from 80 or $90 \mathrm{~K}$ to $4.2 \mathrm{~K}$.

The energy that must be removed during a cool down from $300 \mathrm{~K}$ to $4.2 \mathrm{~K}$ can be calculated using the following expression;

$$
E=M_{S} \int_{T 1}^{T 2} C(T) d T=M_{S}[H(T 2)-H(T 1)]
$$

where $\mathrm{E}$ is the energy removed from a device with a mass $\mathrm{M}_{\mathrm{S}}$ when it is cooled from temperature $\mathrm{T} 2$ to temperature $\mathrm{T} 1$. $\mathrm{C}(\mathrm{T})$ is the average specific heat per unit mass as a function of temperature $\mathrm{T}$. $\mathrm{H}(\mathrm{T} 2)$ is the average enthalpy of the material in the device at temperature $\mathrm{T} 2$, and $\mathrm{H}(\mathrm{T} 1)$ is the average enthalpy of the material at temperature $\mathrm{T} 1$.

Equation 38 can be applied to each of the materials that make up the insertion device. For the purposes of estimating the amount of cryogen needed to cool the device one can assume that the device is made from copper, iron and aluminum. The enthalpy of all of these materials at $4.2 \mathrm{~K}$ is $0.13 \mathrm{~J} \mathrm{~kg}^{-1}$, $0.74 \mathrm{~J} \mathrm{~kg}^{-1}$, and $0.46 \mathrm{~J} \mathrm{~kg}^{-1}$ respectively. At room temperature $(300 \mathrm{~K})$, the enthalpy of these materials is $81100 \mathrm{~J} \mathrm{~kg}^{-1}, 79600 \mathrm{~J} \mathrm{~kg}^{-1}$, and $170400 \mathrm{~J} \mathrm{~kg}^{-1}$ respectively. At a temperature of $90 \mathrm{~K}$, the enthalpy of these materials is $5550 \mathrm{~J} \mathrm{~kg}^{-1}, 8220 \mathrm{~J} \mathrm{~kg}^{-1}$, and $13250 \mathrm{~J} \mathrm{~kg}^{-1}$ respectively. An intermediate temperature of $90 \mathrm{~K}$ was selected because liquid nitrogen cooling can achieve this temperature without leaving liquid nitrogen in the liquid helium tank.

The liquid nitrogen and liquid helium requirements for cool down depend on the insertion device mass and the temperature where one changes from liquid nitrogen cooling to liquid helium cooling. Another factor that comes into play is whether or not the available sensible enthalpy is used in the cool down process [31]. If only the heat of vaporization is used to remove the heat from the insertion device, the mass of cryogen required to cool the device down from temperature $\mathrm{T} 2$ to temperature $\mathrm{T} 2$ takes the following analytic form;

$$
M_{c}=\frac{M_{s}[H(T 2)-H(T 2)]}{h_{f g}}
$$

where $M_{s}$ and $H(T 1)$ and $H(T 2)$ have been defined above. $M_{c}$ is the cryogen mass needed to cool down the device mass $M_{s}$ and $h_{f g}$ is the heat of vaporization of the coolant. (See Table 5 for values of $h_{\mathrm{fg}}$ for nitrogen and helium)

If one want to use the heat of vaporization plus the sensible heat to do the cool down, one can estimate the cryogenic fluid mass needed to do the cool down by using the following expression;

$$
M_{c}=\frac{2 M_{s}[H(T 2)-H(T 2)]}{2 h_{f g}+C_{p}(T 2-T 1)}
$$

$-39 b-$

where $C_{p}$ is the average gas specific heat per unit mass between temperature T1 and T2 (See Table 5 for 
values of $\mathrm{C}_{\mathrm{p}}$ for nitrogen and helium.). When one uses liquid nitrogen as a coolant the mass cryogen calculated for cooling down a device using Equation 40 is about two thirds of that calculated using Equation 39. The reason for this is that the heat of vaporization for nitrogen is $199700 \mathrm{~J} \mathrm{~kg}^{-1}$ where as the heat needed to heat the nitrogen up from $77.3 \mathrm{~K}$ to $300 \mathrm{~K}$ is about $225000 \mathrm{~J} \mathrm{~kg}^{-1}$. With helium, the mass calculated using Equation 40 is less than one tenth of that calculated using Equation 39. The heat of vaporization for helium is only $20300 \mathrm{j} \mathrm{kg}^{-2}$, where as $\mathrm{C}_{\mathrm{p}}=5200 \mathrm{~J} \mathrm{~kg}^{-1} \mathrm{~K}^{-1}$ and the heat needed to heat the helium up from 4.2 to $77.3 \mathrm{~K}$ is about $380000 \mathrm{~J} \mathrm{~kg}^{-1}$. (Note the sensible heat from $4.2 \mathrm{~K}$ to $300 \mathrm{~K}$ for helium is about $1538000 \mathrm{~J} \mathrm{~kg}^{-1}$.) Since liquid helium is expensive, it pays to use its sensible heat as well as its heat of vaporization during a cool down. Since liquid nitrogen is relatively cheap, there is far less to be gained from using the sensible heat along with the heat of vaporization.

The key thing in a cool down using liquid cryogens is when to change from using liquid nitrogen to using liquid helium for the cool down. The answer is a matter of style and experience. If one can be absolutely sure that one can get all of the liquid nitrogen out of the device being cooled, one can cool the device to $77.3 \mathrm{~K}$ before continuing the cool down with liquid helium.

As a rule of thumb, the author suggests that the following procedure be used for cooling with liquid nitrogen; the resistance of the magnet should be measured at room temperature $(300 \mathrm{~K})$ and the magnet resistance should be monitored as the magnet is cooled down. Since the superconductor in the magnet has good copper in the matrix, one can estimate the magnet temperature of the magnet if one knows its resistance with respect to its room temperature resistance. (When the magnet is at $77 \mathrm{~K}$, its resistance has gone down a factor of 7.5.) In order to ensure that no liquid nitrogen ends up in the liquid helium tank, one should stop putting liquid nitrogen into the helium tank when the resistance of the magnet drops about a factor of 5 or 5.5. The final temperature of the magnet and the helium tank will be about $90 \mathrm{~K}$ when one does this.

The second factor is where one puts the liquid into the tank, and where one takes off the boil off gas from the cryostat. The liquid cryogen (nitrogen or helium) should be put into the tank at the bottom at one end. The boil off gas should be taken off the top of the tank at the other end. This will ensure that as much of the magnet heat is taken into the cryogen as sensible heat. The author recommends that even after the tank is filled, that the liquid be added to the tank at the bottom. This will cause mixing of the liquid during a fill, because bubbles will go to the top of the tank. This view is contrary to common practice where the liquid is dumped at the top of a tank during a refill. This is a case where the author thinks that common practice is not always the correct thing to do.

One should start the cool down with liquid helium when the magnet temperature is above $80 \mathrm{~K}$. One should continue to monitor the resistance of the magnet. One should not try to fill the magnet with liquid helium too quickly, because one wants to use as much of the sensible heat that is in the helium gas between the boiling temperature $(4.2 \mathrm{~K})$ and the temperature that the magnet is. Since most of the remaining enthalpy is at magnet temperatures above $40 \mathrm{~K}$, using the sensible heat in the helium gas will save money. If there is a question about the heat transfer to the magnet during the cool down process, perhaps the liquid should be forced through a tube that is thermal connected to the cold mass. With helium, it is really important to use the available sensible heat as well as the latent heat. 
The cooler can be started as one starts the cool down. A typical cooler by itself will get to its operating temperature in about an hour. The cooler cold head will cool down faster than the magnet. So one can have the cooler on while the magnet is being cooled down using liquid. Experience with Superbend and a number of liquid cooled magnets suggest that one should allow up to eight hours to do a magnet cool sown with liquid nitrogen and liquid helium. In general, the more massive the magnet is the longer it will take to cool it down.

When the cryostat start to accept liquid helium, the magnet resistance will change suddenly from about two orders of magnitude below the room temperature resistance to nearly zero as the magnet becomes superconducting. Once the magnet is in the superconducting state, one should see liquid in the helium tank. The final fill can take up to an hour depending on the volume of the helium tank and the amount of heat that remains in the tank and the magnet.

One must remember that the cool down is not finished when the cryostat tanks are filled with helium and nitrogen. At this point the heat leak into the tank is still two or three times the final heat leak that the magnet system is designed for. At this point, the coolers will not be able to keep the load at its desired operating temperature. One should keep filling the cryogen tanks until the magnet stays at its desired operating temperature on the cooler (or coolers) alone. It may take from one to two days for the last little bit of heat to be removed from the insulation. It may take two or three days before the cooler (or coolers) before the magnet can be charged to its design current at the design magnet charging rate.

\section{Insertion Device Cool Down using Small Coolers}

An alternative method for cooling down an insertion device magnet is to use the cooler to do the cooling. This may be an attractive approach if the cooler is connected directly to the load being cooled. If the connection from the cooler to the load is made through a heat pipe, direct cool down using the cooler alone may not work very well.

Experimental evidence from tests of a RDK-415-D cooler [18] suggests that this $1.5 \mathrm{~W}$ (at $4.2 \mathrm{~K}$ ) will deliver over $60 \mathrm{~W}$ at higher temperature, while the first stage delivers over $80 \mathrm{~W}$ above its operating temperature of $60 \mathrm{~K}$. The cooler test for Superbend showed that a load with mass of $25-\mathrm{kg}$ connected to the second stage took about 15 hours to reach its operating temperature of $4.2 \mathrm{~K}$. At the same time a load with a mass of about 22-kg connected to the first stage took about 13 hours to reach its operating temperature. Since the cold mass at $60 \mathrm{~K}$ is less than the cold mass at $4.2 \mathrm{~K}$, one can expect the shields and intercepts to reach operating temperature first. Using the RDK-415-D cooler alone, one can expect the total cool down time to be about 0.6 hours per kilogram of $4 \mathrm{~K}$ cold mass connected to the cooler. The author has not measured the cool down capability of the PT-407 cooler. If one scales the $4 \mathrm{~K}$ performance of the cooler compared to the RDK-415-D cooler, one would expect a total cool down time of about 1.0 to 1.2 hours per kilogram connected to the cooler.

If an insertion device has a mass of $100 \mathrm{~kg}$ and it is cooled by a single RDK-415-D cooler, its cool down time might be 60 hours (2.5 days). If the mass per RDK-415-D cooler is increased to $400 \mathrm{~kg}$, a cool down time of 240 hours might be expected. While one can cool down these devices with their coolers, one probably would use liquid cryogens to do the cool down, because of the time factor. 


\section{Insertion Device Cool Down using a Conventional Refrigerator}

One can use the cold box of a J-T refrigerator to provide the cooling to cool down an insertion device magnet. The cold helium mass flow from the cold box at 20 to $25 \mathrm{~K}$ is proportional to the mass flow of helium in the J-T circuit when the machine is operating at $4.4 \mathrm{~K}$. Figure 19 illustrates how one would cool down an insertion device magnet that normally operates in a bath of liquid helium. Cold gas from the refrigerator goes to the bottom of one end of the bath cryostat. Warm gas leaves the top of the cryostat at the other end and it returns to the refrigerator compressor by-passing the cold box. Once the magnet is cold, the return gas returns to the cold box cooling the J-T heat exchanger, which causes liquid helium to be produced and collected in the magnet cryostat. Phase separation occurs in the magnet cryostat instead of a separate control dewar as is shown in Figure 18.

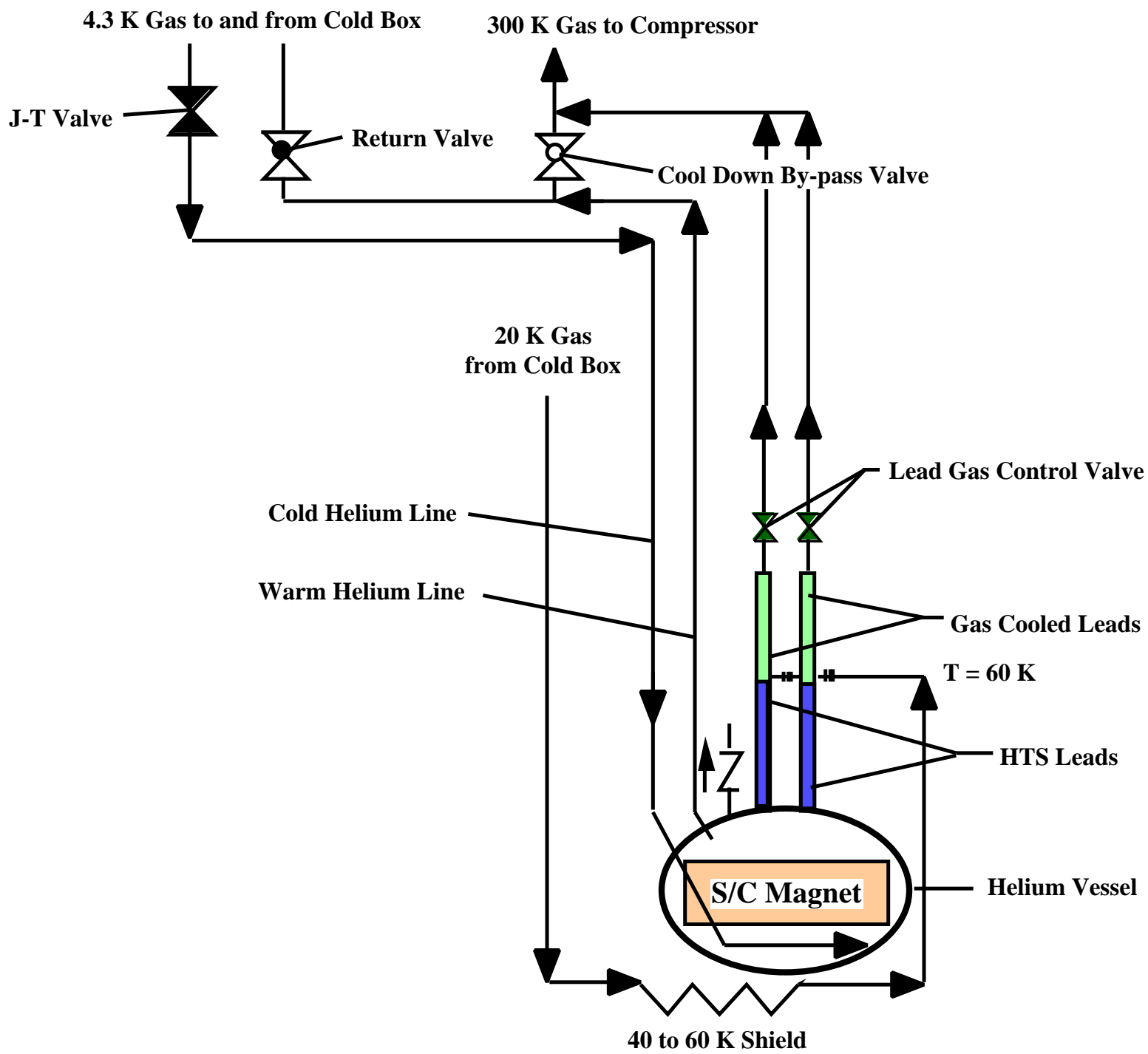

Figure 19. The Cool Down Phase of an Insertion Magnet that is in a Bath Cryostat using a Large J-T Refrigerator (Note: when the magnet is fully cold the by-pass valve is closed and the refrigerator return valve is open. (See Fig. 18)) 
The amount of cold gas produced during the cool down of a magnet depends on the refrigeration capacity once the magnet is cold. In general, large refrigerators deliver (greater than $1 \mathrm{~kW}$ at $4.4 \mathrm{~K}$ ) about $0.00008 \mathrm{~kg} \mathrm{~s}^{-1}$ of helium per watt of $4.4 \mathrm{~K}$ refrigeration delivered at $4.4 \mathrm{~K}$ in the $\mathrm{J}$ - $\mathrm{T}$ circuit during normal operation. Small refrigerators (say $50 \mathrm{~W}$ at $4.4 \mathrm{~K}$ ) are less efficient and they require more mass helium mass flow (up to $0.00012-\mathrm{kg} \mathrm{s}^{-1}$ ) per watt of refrigeration delivered at $4.4 \mathrm{~K}$. When the lower end of the cold box is warm, there is less mass flow in the J-T circuit. During the early phases of the cool down the mass flow through the J-T may be one sixth to one quarter of the mass flow in that circuit during normal operation when there is liquid helium in the cryostat. How much gas can be delivered when the cold box and magnet are warm depends on the design of the cold box heat exchangers.

The cooling power delivered by a refrigerator to the load being cooled can be estimated using the following approximate expression;

$$
R\left(T_{M}\right)=m_{R} C_{p}\left[T_{M}-T_{R}\right]
$$

where $R\left(T_{M}\right)$ is the refrigeration delivered as a function of the gas temperature leaving the magnet $T_{M}$; $\mathrm{m}_{\mathrm{R}}$ is the mass flow of the gas leaving the refrigerator through the J-T circuit at temperature $\mathrm{T}_{\mathrm{R}}$; and $\mathrm{C}_{\mathrm{p}}$ is the specific heat per unit mass of gas leaving the cold box (for helium $\mathrm{C}_{\mathrm{p}}=5200 \mathrm{~J} \mathrm{~kg}^{-1} \mathrm{~K}^{-1}$ ).

If the heat transfer from the magnet to the cold gas circulating around the magnet the temperature of the gas leaving the magnet $\mathrm{T}_{\mathrm{M}}$ will nearly be the same as the warmest part of the magnet. One should design the cool down circuit so that the product of the heat transfer coefficient and the heat transfer area is maximized to that $\mathrm{T}_{\mathrm{N}}$ is very close to the hotest temperature in the magnet.

For a $40 \mathrm{~W}$ refrigerator, the $\mathrm{J}-\mathrm{T}$ circuit mass flow will be in the range of 0.0005 to $0.0011 \mathrm{~kg} \mathrm{~s}^{-1}$ when $T_{M}=275 \mathrm{~K}$. When the gas leaving the refrigerator is at $T_{R}=25 \mathrm{~K}$, the cooling $R\left(T_{M}\right)$ delivered to the insertion magnet will be in the range from 650 to $1450 \mathrm{~W}$. Later in the cool down when the gas leaving the magnet at $\mathrm{T}_{\mathrm{M}}=50 \mathrm{~K}$, the gas flow in the $\mathrm{J}$ - $\mathrm{T}$ circuit is increased maybe 50 percent so the cooling power would range from 100 to $200 \mathrm{~W}$. As the $\mathrm{T}_{\mathrm{M}}$ goes down, the mass flow in the cooling circuit increases such that a $50 \mathrm{~W}$ refrigerator will always deliver more than $50 \mathrm{~W}$ of cooling at magnet temperature above $4.4 \mathrm{~K}$.

As the magnet temperature approaches $10 \mathrm{~K}$ (when a Nb-Ti magnet becomes superconducting), one should close the valve that causes the gas flowing through the magnet to by-pass the cold box return and open the valve to the cold box return. This will cool the J-T heat exchanger enough so that liquid helium will eventually be produced in the magnet cryostat. Since for insertion magnets, the refrigerator may be sized for the leads, there should be enough excess refrigeration to permit one to start making liquid in the magnet cryostat. Once liquid helium starts to collect in the magnet cryostat, the liquid level will rise to a level where liquid helium enters the return pipe to the refrigerator. When the lower heat exchanger of the refrigerator is cooled below its design value the refrigeration production declines. One can speed up the final part of the magnet cool down by adding liquid helium to the dewar and allowing the boil off gas to cool the refrigerator heat exchanger. Whether one can allow the boil off gas to return through the cold box heat exchanger depends on type of expanders that are used in the cold box. 


\section{CONCLUDING COMMENTS}

The performance of a short period insertion device (an undulator pr a wiggler that behaves like an undulator) is strongly dependent on the physical gap between the magnet poles. The performance of the insertion device is also strongly dependent on the current density in the superconducting coils. Both factors will affect how the insertion device is cooled.

A large refrigeration load for an insertion device is magnet bore tube cooling. When the magnet free bore gap is in the range from 8 to $10 \mathrm{~mm}$, a physical bore temperature of 30 to $50 \mathrm{~K}$ appears to be desirable. When the free bore gap is in the 3 to $5 \mathrm{~mm}$ range, cooling the magnet bore to $4.5 \mathrm{~K}$ appears to be desirable. Bore cooling adds to refrigeration load. Large beam currents and small gaps make the bore cooling problem worse. In some of the insertion devices being considered, the cooling of the magnet bore is the largest heat load that will be encountered and this refrigeration load will be a low temperature load.

The insertion device lead current may be affected by the desired overall current density in the superconducting coils. The use of an A-15 conductor such as niobium tin may push one to larger magnet currents (say greater than $2000 \mathrm{~A}$ ). The total current that is put into the insertion magnet through its leads drives the type of refrigerator that is used to cool the device, because cooling for the leads is proportional to the current. Operation of the device in persistent mode will allow one to design the device for less lead cooling. Cooling of the magnet leads is a strong driver in the selection of a refrigerator for an insertion device.

Small cryogenic coolers can be used to cool both the magnet and the magnet bore. There are currently at least three small coolers that are suitable for cooling insertion device magnets. In general, the useful operating range for one or more small coolers is up to 5 watts at $4.2 \mathrm{~K}$ (including the magnet bore). Since small coolers take up heat from the leads in the 60 to $80 \mathrm{~K}$ range, the upper limit for the total lead current is about $4000 \mathrm{~A}$. If the leads are nitrogen cooled, one can go a little higher. Operating the magnet in persistent mode will also help reduce the required lead cooling. Bore tubes at about $40 \mathrm{~K}$ can be cooled using one or two pulse tube coolers. When one has the need for cooling at $4.2 \mathrm{~K}$ that is above $5 \mathrm{~W}$ and one has high total lead currents, one must use a conventional refrigerator to do the cooling. There are several suitable candidate conventional refrigerators that can be used.

The connection of a small cooler to the device being cooled is critical, particularly at $4.2 \mathrm{~K}$. If one makes the connection from a small cooler to the load using a copper strap, the RRR of the strap must be high, the length of the strap must be short, and the strap cross-section area must be large enough to ensure that the $\Delta \mathrm{T}$ between the load and the cooler is less than $0.2 \mathrm{~K}$. The parameters of the copper strap are less important when they are used in the 50 to $80 \mathrm{~K}$ range. Helium heat pipes are a good way of ensuring that the $\Delta \mathrm{T}$ between the load and the cold head is less than $0.1 \mathrm{~K}$. Heat pipes permit the

cooler to be up to a meter from the load. In addition, heat pipes can act like a thermal diode so that the heat flow from the cold head to the magnet will be low when the magnet is colder than the cooler. 


\section{ACKNOWLEDGEMENTS}

The author acknowledges discussions with Soren Prestemon, Ross Schlueter, John Corlett, and others at the Lawrence Berkeley National Laboratory concerning absorber various aspects of wiggler and undulator design. This work was supported by the Office of Science, United States Department of Energy under DOE contract number DE-AC03-76SF00098

\section{REFERENCES}

[1] M. A. Green, E. H. Hoyer, R. D. Schlueter et al, "Refrigeration Options for the Advanced Light Source Superbend Dipole Magnets," Advances in Cryogenic Engineering 45 p 1363, Plenum Press, New York (2000)

[2] "Review of Particle Properties," Reviews of Modern Physics 48, Number 2, Part II, April 1976

[3] John D. Jackson, Classical Electrodynamics, third Edition, John Wiley and Sons Inc, New York

[4] Center for X-ray Optics, Advance Light Source, "X-Ray Data Booklet," second Edition, Lawrence Berkeley National Laboratory, January 2001

[5] M. A. Green, "Heat Transfer through a Multi-layer Insulation System as a Function of Pressure in the Cryostat Vacuum Space," Advances in Cryogenic Engineering 43, p 1313, Plenum, (1997)

[6] I. A. Black, P. E. Glaser, "Effects of Compressive Loads in the Heat Flux through Multilayer Insulations," Advances in Cryogenic Engineering 11, p 26, Plenum Press, New York (1965)

[7] M. A. Green, R. A. Byrns and S. J. St Lorant, "Estimating the Cost of Superconducting Magnets and the Refrigerators Needed to Keep Them Cold," Advances In Cryogenic Engineering 37, p 637, Plenum Press, New York, 1991

[8] M. A. Green and S. J. St Lorant, "Estimating the Cost of Large Superconducting Thin Solenoid Magnets," Advances in Cryogenic Engineering 39, p 271-276, Plenum Press, New York, 1993

[9] D. B. Hopkin's, "Bunched Beam I ${ }^{2}$ R Beam Pipe Dissipation," a single graph that was issued on July 7, 1974 for ESCAR

[10] M. A. Green, "ESCAR Refrigeration, Bunched Beam and Eddy Current Losses in a ESCAR Bore Tube," Lawrence Radiation Laboratory Engineering Note M-4747, (UCID-3687) 6 September 1974.

[11] W. Chou and F. Ruggiero, "Anomalous Skin Effect and Resistive Wall Heating," CERN-LHC Note 2, 8 September 1995.

[12] A. B. Pippard, Proceedings of the Royal Society (London), A191, p 385 (1947)

[13] B. V. Proobedov, private communication concerning extreme anomalous skin effect heating versus normal skin effect heating, 19 February 2003

[14] Martin M. Wilson, Superconducting Magnets, pp 256 - 272, Oxford Science Publications, Clarendon Press, Oxford UK, (1983)

[15] J. Zbasnik, S. T. Wang, J. Y. Chen, et al, "ALS Superbend Magnet System", IEEE Transactions on Applied Superconductivity 11, p 2531, (2001) 
[16] M. A. Green, "The Effect of Low Temperature Cryo-coolers on the Development of Low Temperature Superconducting Magnets," IEEE Transactions on Applied Superconductivity 11, No. 2, p 2615, (2001)

[17] The Sumitomo Data Sheet for the SRDK-415D Cryo-cooler from Janis Research Inc. Willington MA 01887 (Janis is the US seller of Sumitomo cryo-coolers.)

[18] J. Zbasnik, M. A. Green E H. Hoyer et al "Tests of a GM Cryo-cooler and High Tc Leads for the ALS Superbend Magnets," Advances in Cryogenic Engineering 45 p 635, Plenum Press (1999)

[19] P. Gifford and C. Wang, Cryomech Inc, Syracuse NY 13211, private communication concerning the PT-405 pulse tube cryo-cooler and its performance (August 2000)

[20] Cryomech Cryorefrigerator Specifications, Cryomech Inc., Syracuse NY 13211, (2003), see the Cryomech web site at www.cryomech.com

[21] C. E. Taylor, C. M. Lyneis, D. Leitner, and S. Abbott, "An Efficient Cooling Loop for Connecting a Cryo-cooler to a Liquid Helium Reservoir," to be presented at the Cryogenic Engineering Conference, Anchorage Alaska, 22 through 26 September 2003.

[22] C. Taylor, "Design of a Helium Re-condenser for the Venus Cryostat," LBNL Superconducting Magnet Group Report 759, January 2002

[23] M. A. Green, "The Integration of a Liquid Cryogen Cooling and Cryo-coolers with Superconducting Electronic Systems," to be published by Superconducting Science and Technology, ISEC-2003 Special Issue,

[24] Frank Kreith, Principles of Heat Transfer, International Text Book Co., Scranton PA, (1958)

[25] Victor J. Johnson Ed., A Compendium of the Properties of Materials at Cryogenic Temperature, Pergamon Press, New York (1961)

[26] T. K. H. Frederking, "Peak Heat Flux and Temperature Difference in Nucleate Boiling of Liquefied Gases," Advances in Cryogenic Engineering 8, p 489, Plenum Press, New York, (1962)

[27] E. G. Bretari and R. V. Smith, "Nucleate and Film Pool Boiling Design Correlations for $\mathrm{O}_{2}, \mathrm{~N}_{2}$, $\mathrm{H}_{2}$, and He," Advances in Cryogenic Engineering 10, p 325, Plenum Press, New York, (1964)

[28] D. N. Lyon, "Boiling Heat Transfer and Peak Nucleate Boiling Fluxes in Saturated Liquid Helium between the L and Critical Temperatures," Advances in Cryogenic Engineering 10, p 371, Plenum, New York, (1964)

[29] M. A. Green, et al., "Operation of a Forced Two-phase Cooling System on a Large Superconducting Magnet," Proceedings of the International Cryogenic Engineering Conference 8, Genoa Italy, p 72, Butterworth Press, Oxford UK, (1980)

[30] M. A. Green, "Design Parameters for the g-2 Solenoid and Inflector Magnet Two-phase Cooling System," Brookhaven National Laboratory g-2 Note 154, 29 April 1993

[31] R. B. Jacobs, Liquid Requirements for the Cool-down of Cryogenic Equipment," Advances in Cryogenic Engineering 8, p 529, Plenum, New York, (1962) 


\section{DISCLAIMER}

This document was prepared as an account of work sponsored by the United States Government.

While this document is believed to contain correct information, neither the United States

Government nor any agency thereof, nor The Regents of the University of California, nor any of their employees, makes any warranty, express or implied, or assumes any legal responsibility for the accuracy, completeness, or usefulness of any information, apparatus, product, or process disclosed, or represents that its use would not infringe privately owned rights. Reference herein to any specific commercial product, process, or service by its trade name, trademark, manufacturer, or otherwise, does not necessarily constitute or imply its endorsement, recommendation, or favoring by the United States Government or any agency thereof, or The Regents of the University of California. The views and opinions of authors expressed herein do not necessarily state or reflect those of the United States Government or any agency thereof, or The Regents of the University of California.

Ernest Orlando Lawrence Berkeley National Laboratory is an equal opportunity employer. 